\title{
Topologies and Bornologies Determined by Operator Ideals
}

\author{
Yau-Chuen Wong and Ngai-Ching Wong \\ Department of Mathematics, The Chinese University of Hong Kong, Shatin, N.T., Hong kong
}

\section{Introduction}

The notion of topologies, introduced by Stephani[10], is useful for studying the injective hull of an operator ideal. Using Randtke's idea(see [8,p.90] or [12,(3.2.1)and(3.2.7)]), we can characterize generating topologies in terms of seminorms which satisfy some expected properties(see Lemma3.3 and Theorems3.4 and 3.9). By a well-known and useful ideal of Grothendieck, the dual notions of generating topologies and ideal-topologies, the so-called generating bornologies, are given and studied in Sect.4. In terms of ideal-bornogies, the surjective hull of an operator ideal on Banach spaces is given(see Lemma4.8 and Theorem4.10). In terms of these two dual concepts, we are able to classify locally convex spaces, and to study their dual results. For instance, we show that if $\mathfrak{A}$ is a symmetric(resp.completely symmetric) operator ideal on Bnanch spaces then a Banach space $E$ is an $\mathfrak{A}$-topological space( $\mathfrak{A}$-bornological space) if and only if its Banach dual space $E^{\prime}$ is $\mathfrak{A}$-bornological(resp. $\mathfrak{A}$-topological)(Theorem 5.9). Also we are able to define the most natural and the most applicable type of operator ideals on $L C S^{\prime} s$, namely the $\mathfrak{G}-\mathfrak{B}$-operators. This is an extension of the notions of quasiSchwartz operators defined by Randtke[8,p.91] and of cone-prenuclear maps defined by Wong[12,p.142]. Sufficient conditions are given to ensure that the $\mathfrak{G}-\mathfrak{B}$-operators from an injective(resp.surjective) operator ideal(see Propositions 6.3 and 6.4). Finally, we point out that a formula concerning with the injective hull of a bounded operator ideal, given by Franco and Piñeiro[3, Theorem 1 in Sect.2], is not true(see Example 6.6) 


\section{Preliminary Results of Operator Ideals and Bornolo- gies}

Throughout this paper, the class of all locally convex (Hausdorff) spaces(abbreviated by $L C S^{\prime} s$ )(resp.all Banach spaces) is denoted by $\mathbb{L}($ resp. $\mathbb{B})$, the class of all operators (i.e. continuous linear maps) between arbitrary locally convex spaces is denoted by $\mathfrak{L}$, while $\mathfrak{L}(X, Y)$ stands for the vector space of all operators from $\mathrm{X}$ into $\mathrm{Y}$ (where $\mathrm{X}, \mathrm{Y} \in \mathbb{L}$ ), and $F_{\text {ori }}(X)$ denotes the original locally convex topology on $X \in \mathbb{L}$. For any subclass $b$ of $\mathbb{L}$, we write

$$
\mathfrak{L}_{l}=U\{\mathfrak{L}(X, Y): X, Y \in b\} .
$$

For any $\mathrm{E} \in \mathbb{B}, E^{\prime}$ stands for the Banach dual of $\mathrm{E}, U_{E}$ denotes the closed unit ball in $\mathrm{E}$, and the norm-topology on $\mathrm{E}$ is denoted by $\|\cdot\|_{E}$-topology, also we put

and dually

$$
E^{i n j}=l_{\infty}\left(U_{E^{\prime}}\right) \quad \text { and } \quad J_{E^{x}}=\left[\left\langle x, u^{\prime}\right\rangle\right]_{u^{\prime} \in U_{E^{\prime}}} \quad(x \in E)
$$

$$
E^{\text {sur }}=l_{1}\left(U_{E}\right) \quad \text { and } \quad Q_{E}\left(\left[\xi_{x}\right]_{x \in U_{E}}\right)=\sum_{U_{E}} \xi_{x} \cdot x \quad\left(\left[\xi_{x}\right]_{x \in U_{E}} \in l_{1}\left(U_{E}\right)\right) .
$$

Let 1 be a sugclass of $\mathbb{L}$ containing $\mathbb{K}$. Following Pietsch[7], we call a subclass $\mathfrak{A}$ of $\Omega_{l}$ an operator ideal on 1 if the components

$$
\mathfrak{A}(X, Y)=\mathfrak{A} \cap \mathfrak{L}(X, Y)(X, Y \in \ell)
$$

satisfy the following conditions:

$\left(O I_{0}\right) i d_{\mathbb{K}} \in \mathfrak{A}(\mathbb{K}, \mathbb{K})($ where $\mathbb{K}$ is either $\mathbb{R}$ or $\mathbb{C})$

$\left(O I_{1}\right) \mathfrak{A}(X, Y)$ is a vector subspace of $\mathfrak{L}(X, Y)(X, Y \in b)$

$\left(O I_{2}\right)$ If $S \in \mathfrak{L}\left(X_{0}, X\right), T \in \mathfrak{A}(X, Y)$ and $R \in \mathfrak{L}\left(Y, Y_{0}\right)$ then $R T S \in \mathfrak{L}\left(X_{0}, Y_{0}\right)$

(whenever $X, X_{0}, Y, Y_{0} \in b$ )

In particular, if $b=\mathbb{B}$ (resp. $\mathbb{L}$ or the class of all bornological spaces) then it is called an operator ideal on B-spaces(resp. on LCS's or on bornological spaces).

The class $\Omega$ of all operators is the greatest operator ideal while the class $\mathfrak{F}$ of all operator with finite ranks is the smallest one; also the class of all compact(resp. weakly compact, completely continuous) operators is an operator ideal on $\mathbb{L}$. Recall that an operator $T \in \Omega(X, Y)$ (where $X, Y \in \mathbb{L}$ ) is bounded if T sends some 0-neighbourhood in X onto a bounded set in $Y$. The class of all bounded operators between arbitrary locally convex 
spaces, denoted by $\mathfrak{L}$, is an operator ideal on LCS's.

Let $\mathfrak{A}$ be an operator ideal on b. We say that $\mathfrak{A}$ is:

(a) injective if for all spaces $X, Y \in b$, any operator $T \in \mathfrak{L}(X, Y)$ belongs to $\mathfrak{A}(X, Y)$ if there is some $Z \in b$ and some topological injection(i.e., an one-to-one and relatively open) $J: Y \rightarrow Z$ such that

$$
J T \in \mathfrak{A}(X, Z)
$$

(b) surjective if for all space $X, Y \in b$, any operator $T \in \mathfrak{L}(X, Y)$ belongs to $\mathfrak{A}(X, Y)$ if there is some $G \in b$ and a topological surjection(i.e., an open operator) $Q: G \rightarrow X$ such that

$$
T Q \in \mathfrak{A}(G, Y) .
$$

The injective hull(resp.surjective hull) of an operator ideal $\mathfrak{A}$ on $b$, denoted by $\mathfrak{A}^{i n j}\left(\right.$ resp. $\left.\mathfrak{A}^{\text {sur }}\right)$, is defined as the intersection of all injective (resp. surjective) operator ideals on $b$ containing $\mathfrak{A}$.

If $\mathfrak{A}$ is an operator ideal on B-spaces, then $\mathfrak{A}^{\text {inj }}$ and $\mathfrak{A}^{\text {sur }}$ can be represented simply as follows:

$$
\mathfrak{A}^{i n j}(E, F)=\left\{T \in \mathfrak{L}(E, F): J_{F} T \in \mathfrak{A}\left(E, F^{i n j}\right)\right\}
$$

and

$$
\mathfrak{A}^{\text {sur }}(E, F)=\left\{T \in \mathfrak{L}(E, F): T Q_{E} \in \mathfrak{A}\left(E^{\text {sur }}, F\right)\right\},
$$

whenever $\mathrm{E}$ and $\mathrm{F}$ are B-spaces. Moreover, we have the following remarkable characterization for $\mathfrak{A}^{i n j}$ and $\mathfrak{A}^{\text {sur }}$ due to Stephani(see[7,p.109 and p.112]).

Lemma 2.1 Let $\mathfrak{A}$ be an operator ideal on $B$-spaces and $T \in \mathfrak{L}(E, F)$, where $E$ and $F \in \mathbb{B}$.

(a) $T \in \mathfrak{A}^{i n j}(E, F)$ if and only if there exists an $F_{0} \in \mathbb{B}$ and an $S \in \mathfrak{A}\left(E, F_{0}\right)$ such that

$$
\|T x\| \leqq\|S x\| \quad(\text { for all } x \in E)
$$

(b) $T \in \mathfrak{A}^{\text {sur }}(E, F)$ if and only if there exists an $E_{0} \in \mathbb{B}$ and an $R \in \mathfrak{A}\left(E_{0}, F\right)$ such that

$$
T U_{E} \subseteq R U_{E_{0}}
$$

It is natural to ask whether $(2 \mathrm{a})$ and $(2 \mathrm{~b})$ can be extended to the case of an operator ideal $\mathfrak{A}$ on LCS's. To do this, we require the following construction, due to Franco and 
Pi $\tilde{n}$ eiro[3].

Given a locally convex space X, let $E\left(X^{\prime}\right)$ be the class of all $\sigma\left(X^{\prime}, X\right)$-closed, absolutely convex, equicontinuous subsets of $X^{\prime}$, and let $X^{i n j}$ be the product space of a family $\left\{l_{\infty}(D): D \in E\left(X^{\prime}\right)\right.$ of B-spaces, i.e.,

$$
X^{i n j}=\prod\left\{l_{\infty}(D): D \in E\left(X^{\prime}\right) .\right.
$$

Let us define $J_{x}: X \rightarrow X^{i n j}$ by setting

$$
J_{X}(x)=\left[J_{X, D}(x)\right]_{D \in E\left(X^{\prime}\right)},
$$

where $J_{X, D}(x)$ is a bounded function on $\mathrm{D}$ with values

$$
J_{X, D}(x)\left(d^{\prime}\right)=\left\langle x, d^{\prime}\right\rangle \quad\left(\text { for all } d^{\prime} \in D\right) .
$$

Lemma 2.2 (Franco and Pr̃neiro[3]). Let $\mathfrak{A}$ be an operator ideal on LCS's. Then the injective hull of $\mathfrak{A}$ is given by

$$
\mathfrak{A}^{i n j}(X, Y)=\left\{T \in \mathfrak{L}(X, Y): J_{Y} T \in \mathfrak{A}\left(X, Y^{i n j}\right)\right\}, \quad(X, Y \in \mathbb{L}) .
$$

Let $\mathrm{X}$ be a vector space over $\mathbb{K}$. Following Hogbe-Nlend[4], by a vector bornology on $\mathrm{X}$ we mean a family $\mathfrak{B}$ of subsets of $\mathrm{X}$ satisfies the following conditions:

$\left(V B_{1}\right) \quad X=\cup \mathfrak{B}$

$\left(V B_{2}\right)$ if $B \in \mathfrak{B}$ and $A \subseteq B(A \subseteq X)$ then $A \in \mathfrak{B}$;

$\left(V B_{3}\right) B_{1}+B_{2} \in \mathfrak{B}$ whenever $B_{1}, B 2 \in \mathfrak{B}$;

$\left(V B_{4}\right) \lambda B \in \mathfrak{B}$ whenever $\lambda \in \mathbb{K}$ and $B \in \mathfrak{B}$;

$\left(V B_{5}\right)$ the circle hull of any $B \in \mathfrak{B}$ belongs to $\mathfrak{B}$.

Elements in $\mathfrak{B}$ are called $\mathfrak{B}$-bounded sets in X.

A vector bornology $\mathfrak{B}$ on $\mathrm{X}$ is called a convex bornology if

$$
\Gamma B \in \mathfrak{B} \text { for all } B \in \mathfrak{B},
$$

where $\Gamma B$ is the absolutely convex hull of $\mathrm{B}$. The pair $(X, \mathfrak{B})$ is called a convex bornological space which is denoted by $X^{\mathfrak{B}}$.

A base of a vector bornology $\mathfrak{B}$ on $X$ is any subfamily $\mathfrak{B}_{0}$ of $\mathfrak{B}$ such that any element in $\mathfrak{B}$ is contained in some member in $\mathfrak{B}_{0}$. It is not hard to show that a collection $\mathfrak{U}$ 
consisting of subsets of $\mathrm{X}$ is a base for a vector bornology(resp. convex bornology) if and only if $\mathfrak{U}$ satisfies the following conditions:

(i) $X=\cup \mathfrak{U}$

(ii) for any $B_{1}, \ldots, B_{n} \in \mathfrak{U}$, there is an $B \in \mathfrak{U}$ such that $B_{1}+\ldots+B_{n} \subseteq B$;

(iii) for any $B \in \mathfrak{U}$ and $\lambda \in \mathbb{K}$, there is an $M \in \mathfrak{U}$ such that $\lambda B \subseteq M$;

(iv) the circled(resp. absolutely convex) hull of any $A \in \mathfrak{U}$ is contained in some member of $\mathfrak{U}$.

If $\mathfrak{U}$ is a base, then the family $\mathfrak{B}(\mathfrak{U})$, defined by

$$
\mathfrak{B}(\mathfrak{U})=\{A \subseteq X: A \subseteq B \text { for some } B \in \mathfrak{U}\},
$$

is a vector(resp. convex) bornology on $\mathrm{X}$ with $\mathfrak{U}$ as a base. $\mathfrak{B}(\mathfrak{U})$ is called the vector (resp. convex) bornology generated by $\mathfrak{U}$.

Let $\mathfrak{U}$ and $\mathfrak{R}$ be two bases for two vector bornologies on $\mathrm{X}$. We say that $\mathfrak{U}$ is coarser than $\mathfrak{R}$, denoted by $\mathfrak{U} \leqq \Re$ if

$$
\mathfrak{B}(\mathfrak{R}) \subseteq \mathfrak{B}(\mathfrak{U})
$$

This is equivalent to that every element in $\mathfrak{R}$ is contained in some members of $\mathfrak{U}$.

We list some important vector bornologies on a locally convex space $\left(X, \mathfrak{T}_{\text {ori }}(X)\right)$ as follows:

(a) the von Neumann bornology on $\mathrm{X}$, denoted by $\mathfrak{U}_{\text {von }}(X)$, is defined to be the class of all bounded sets in $\mathrm{X}$;

(b) the precompact bornology on $\mathrm{X}$, denoted by $\mathfrak{U}_{p c}(X)$, is the class of all $\mathfrak{T}_{\text {ori }}(X)$ precompact sests in $\mathrm{X}$;

(c) the compact bornology on $\mathrm{X}$, denoted by $\mathfrak{U}_{c}(X)$, is the class of all $\mathfrak{T}_{\text {ori }}(X)$-compact sets in $\mathrm{X}$;

(d) the weakly compact bornology, denoted by $\mathfrak{U}_{w c}(X)$, is the class of all $\sigma\left(X, X^{\prime}\right)$ compact subsets of $\mathrm{X}$;

(e) the finitely dimensional bornology, denoted by $\mathfrak{U}_{f}(X)$, is defined to be the class of all subsets $\mathrm{A}$ of $\mathrm{X}$ satisfying 


$$
A \subseteq \Gamma\left(\left\{x_{1}, x_{2}, \ldots, x_{n}\right\}\right)
$$

for some finite subset $\left\{x_{1}, x_{2}, \ldots x_{n}\right\}$ of $\mathrm{X}$.

Let $(X, \mathfrak{T})$ be a locally convex space. For any subset B of $\mathrm{X}$, the $\sigma$-disked hull of $\mathrm{B}$ is defined by

$$
\Gamma_{\sigma} B=\left\{\sum_{n=1}^{\infty} \lambda_{n} b_{n}: \sum_{n=1}^{\infty}\left|\lambda_{n}\right| \leqq 1, \lambda_{n} \in \mathbb{K}, b_{n} \in B(n=1,2, \ldots)\right\}
$$

A set $\mathrm{B}$ in $\mathrm{X}$ is said to be $\sigma$-disked if $B=\Gamma_{\sigma} B$.

An absolutely convex, bounded subset $\mathrm{B}$ of $\mathrm{X}$ is said to be infracomplete(or a Banach disk) if the normed space $\left(X(B), r_{B}\right)$ is complete, where

$$
X(B)=\bigcup_{n \geqq 1} n B
$$

and $r_{B}$ is the gauge of $\mathrm{B}$ defined on $\mathrm{X}(\mathrm{B})$. The image of an infracomplete (bounded, absolutely convex) set under an operator is infracomplete.

Lemma 2.3 Let $(X, \mathfrak{P})$ be a locally convex space and $B$ an absolutely convex bounded subset of $X$.

(a) If $B$ is $\sigma$-disked then $B$ is infracomplete.

(b) If $B$ is infracomplete and closed then $B$ is $\sigma$-disked.

Proof.(a)The mapping $Q_{B}: l_{1}(B) \rightarrow X$, defined by

$$
Q_{B}\left(\left[\xi_{x}\right]_{x \in B}\right)=\sum_{x \in B} \xi_{x} \cdot x
$$

is an operator with

$$
Q_{B}\left(U_{l_{1}(B)}\right)=\Gamma_{\sigma} B
$$

As $\left(U_{l_{1}(B)}\right)$ is infracomplete in the B-space $l_{1}(B)$, it follows from $B=\Gamma_{\gamma} B$ that $\mathrm{B}$ is infracomplete.

(b)Follows from the fact that $\mathrm{B}$ is the closed unit ball in the B-space $\left(X(B), r_{B}\right)$.

Let $\mathfrak{B}(X)$ and $\mathfrak{B}(Y)$ be vector bornologies on $\mathrm{X}$ and $\mathrm{Y}$ resp. A linear map $T: X \rightarrow$ $Y$ is said to be locally bounded if

$$
T(B) \in \mathfrak{B}(Y) \quad \text { for all } \quad B \in \mathfrak{B}(X) .
$$

The set of all locally bounded maps from $(X, \mathfrak{B}(X))$ into $(Y, \mathfrak{B}(Y))$, denoted by $L^{x}(X, Y)$, is a vector space. In particular, we write

$$
X^{x}=L^{x}(X, \mathbb{K})
$$


and called the bornological dual of $(X, \mathfrak{B}(X))$.

Let $\left\{\left(X_{i}, \mathfrak{B}_{i}\right): i \in \Lambda\right\}$ be a family of vector(resp. convex) bornological spaces, let $\mathrm{Y}$ be a vector space and let $T_{i}: X_{i} \rightarrow Y$ be linear. For any $i \in \Lambda$, let

$$
T_{i}\left(\mathfrak{B}_{i}\right)=\left\{T_{i}(A): A \in \mathfrak{B}_{i}\right\} .
$$

It is clear that the intersection of a family of vector(resp. convex) bornologies is a vector(resp. convex) bornology. Thus the intersection of all vector(resp. convex) bornologies containing the family $\cup_{i \in A} T_{i}\left(\mathfrak{B}_{i}\right)$ is called the final vector(resp. convex) bornology on $Y$ for the maps $T_{i}$, and is denoted by $\mathfrak{U}(Y)$. It is easily seen that $\mathfrak{U}(Y)$ is the finest vector(resp. convex) bornology on $\mathrm{Y}$ for which all the maps $T_{i}$ are locally bounded.

Throughout this paper, terminology and notation concerning operator ideals will follow Pietsch[7] and Junek[5], while Schaefer[9] and Köthe[6] will serve as our reference for material vector spaces. The background material concerning bornologies can be found in Hogbe-Nlend[4].

\section{Ideal-Topologies and Generating Topologies}

In the sequel, $\mathfrak{b}$ will be assumed to be a subclass of $\mathbb{L}$ containing all normed spaces, the finite product spaces of members in $\mathfrak{b}$ and subspaces of members in $\mathfrak{b}$.

It is clear that the operator ideal $\mathfrak{F}$ on $\mathbb{L}$ (consisting of operators with finite ranks) is injective; moreover, we have, for any $X \in \mathbb{L}$ and any normed space $\mathrm{F}$, that

$$
\mathfrak{F}(X, F)=\mathfrak{L}\left(X_{\sigma}, F\right),
$$

where $X_{\sigma}=\left(X, \sigma\left(X, X^{\prime}\right)\right)$.

Let $\left(E, E_{+}, \mathfrak{T}\right)$ be a locally solid space(for definition, see[12, p.29]) and Y a locally convex space. An $T \in \mathfrak{L}(E, Y)$ is cone-absolutely summing if for any continuous seminorm $\mathrm{q}$ on $\mathrm{Y}$ there exists a continuous seminorm $\mathrm{p}$ on $\mathrm{E}$ such that

$$
\sum_{i=1}^{n} q\left(T u_{i}\right) \leqq p\left(\sum_{i=1}^{n} u_{i}\right)
$$

holds for finite subset $\left\{u_{1}, \ldots, u_{n}\right\}$ of $E_{+}$. Denoted by $\mathfrak{L}^{l}(E, Y)$ the vector space of all cone-absolutely summing operators. Then one can show(see[12,(3.1.3)]) that

$$
\mathfrak{L}^{l}(E, Y)=\mathfrak{L}\left(E_{\sigma_{s}}, Y\right)
$$

where $E_{\sigma_{s}}=\left(E, E_{+}, \sigma_{s}\left(E, E^{\prime}\right)\right)$ and $\sigma_{s}\left(E, E^{\prime}\right)$ is the topology on $\mathrm{E}$ of uniform convergence on all intervals [-f,f] with $f \in E_{+}$(i.e., the locally solid topology associated with $\left.\sigma\left(E, E^{\prime}\right)\right)$.

It is remarkable that (3a) [resp.(3b)] shows that the vector space $\mathfrak{F}(X, F)\left[\operatorname{resp} . \mathfrak{L}^{l}(E, Y)\right]$ 
does not depend upon the original locally convex topology $\mathfrak{T}_{\text {ori }}(X)$ on $\mathrm{X}$ (resp. the original locally solid topology on $\mathrm{E})$, but only on the dual pair $<X, X^{\prime}>$ (resp. $<E, E^{\prime}>$ ). Also, (3a) and (3b) suggest naturally the following:

(Q1)Let $\mathfrak{A}$ be a given operator ideal on $\mathfrak{b}$. For any $X \in \mathfrak{b}$, does there exist a (compactible) locally convex topology $\mathfrak{P}\left(\right.$ w.r.t $<X, X^{\prime}>$ ) such that

$$
\mathfrak{A}(X, Y)=\mathfrak{L}\left(X_{\mathfrak{P}}, Y\right) \quad(\text { for all } Y \in \mathfrak{b}) ?
$$

Conversely, it is natural to ask the following:

(Q2)What kind of compatible locally convex topologies $\mathfrak{P}$ will ensure that the setting

$$
\mathfrak{A}[\mathfrak{P}](X, Y)=\mathfrak{L}\left(X_{\mathfrak{P}}, Y\right) \quad(\text { for all } X, Y \in \mathfrak{b})
$$

defines an operator ideal $\mathfrak{A}[\mathfrak{P}]$ on $\mathfrak{b}$ ?

In order to answer the question (Q1), we require the following:

Definition 3.1 Let $\mathfrak{A}$ be an operator ideal on $\mathfrak{b}$ For any given $X \in \mathfrak{b}$, let $\mathfrak{P}(\mathfrak{A})(X)$ be the projective topology on $\mathrm{X}$ with respect to the family

$$
\left\{\left(Y, \mathfrak{T}_{\text {ori }}(Y), T\right):\left(Y, \mathfrak{T}_{\text {ori }}(Y)\right) \in \mathfrak{b} \quad \text { and } \quad T \in \mathfrak{A}(X, Y)\right\}
$$

The collection, defined by

$$
\mathfrak{P}(\mathfrak{A})=\{\mathfrak{P}(\mathfrak{A})(X): X \in \mathfrak{b}\},
$$

is called the $\mathfrak{A}$-topology (or ideal-topology) on $\mathfrak{b}$. On each $X \in \mathfrak{b}, \mathfrak{P}(\mathfrak{A})(X)$-continuous seminorms on $\mathrm{X}$ are referred to as $\mathfrak{A}$-seminorms(or ideal-seminorms) on $\mathfrak{b}$. We write $X_{\mathfrak{P}(\mathfrak{A})}$ for $(X, \mathfrak{P}(\mathfrak{A})(X))$. The definition of projective topologies implies that

$$
\mathfrak{P}(\mathfrak{A})(X) \leqq \mathfrak{T}_{\text {ori }}(X) \quad \text { and } \quad \mathfrak{A}(X, Y) \subseteq \mathfrak{L}\left(X_{\mathfrak{P}(\mathfrak{A})}, Y\right)
$$

whenever $X, Y \in$ scriptb. As $\mathfrak{F}(X, Y) \subseteq \mathfrak{A}(X, Y)$, it follows that $X^{\prime} \subseteq(X, \mathfrak{P}(\mathfrak{A})(X))^{\prime}$, and hence that $\mathfrak{P}(\mathfrak{A})(X)$ is consistent with $\left\langle X, X^{\prime}\right\rangle$. Also, the definition of projective topologies implies that the ideal-topology $\mathfrak{P}(\mathfrak{A})$ on $\mathfrak{b}$ is determined by the family $\mathfrak{l}(\mathfrak{A})=\{\mathfrak{l}(\mathfrak{A})(X): X \in \mathfrak{b}\}$ of seminorms, where

$$
\begin{aligned}
\mathfrak{l}(\mathfrak{A})(X)=\{ & \left\{q_{Y} \circ S: S \in \mathfrak{A}(X, Y), Y \in \sigma b\right. \text { and } \\
& \left.q_{Y} \text { is } \mathfrak{T}(Y) \text {-continuous seminorms }\right\} .
\end{aligned}
$$

It then follows that a seminorm $\mathrm{p}$ on $\mathrm{X}$ is an $\mathfrak{A}$-seminorm if and only if there exits an $Z \in \mathfrak{b}$, an $S \in \mathfrak{A}(X, Z)$ and a $\mathfrak{T}_{\text {ori }}(Z)$-continuous seminorm $r_{Z}$ on $Z$ such that

$$
p(x) \leqq r_{Z}(S x) \quad(\text { for all } x \in X),
$$


and hence that $T \in \mathfrak{L}\left(X_{\mathfrak{P}(\mathfrak{A}), Y}\right)$ if and only if for any $\mathfrak{T}_{\text {ori }}(Y)$-continuous seminorm $q_{Y}$ on $\mathrm{Y}$, there exists an $Z \in \mathfrak{b}$, an $S \in \mathfrak{A}(X, Z)$ and an $\mathfrak{T}_{\text {ori }}(Z)$-continuous seminorm $r_{Z}$ on $\mathrm{Z}$ such that

$$
q_{Y}(T x) \leqq r_{Z}(S x) \quad(\text { for all } x \in X)
$$

Applying(3e) to the case of Banach spaces, we obtain immediately from Lemma 2.1(a) the following:

Lemma 3.2 Let $\mathfrak{A}$ be an operator ideal on B-spaces. Then

$$
\mathfrak{L}\left(E_{\mathfrak{P}(\mathfrak{A}), F}\right)=\mathfrak{A}^{i n j}(E, F) \quad \text { for all } E, F \in \mathbb{B} .
$$

Note. Formula(3.2.1) is no longer true for an operator ideal on $\mathfrak{b}$ with $\mathfrak{b} \neq \mathbb{B}$ [see Theo$\operatorname{rem}(3.8)(\mathrm{a})]$.

The preceding result was mentioned by Stephani[11, p.242].

In order to give a characterization of seminorms which are $\mathfrak{A}$-seminorms, we require the following notation. Let $\mathrm{X}$ be a locally convex space. For any seminorm $\mathrm{p}$ on $\mathrm{X}$ we write $X_{p}$ for the quotient space $X / p^{-1}(0)$ equipped with the quotient seminorm $\hat{p}\left(\right.$ or $\left.\|\cdot\|_{p}\right)$ of $\mathrm{p}(\hat{p}$ is actually a norm $), \tilde{X}_{p}$ for the completion of $X_{p}$ and $Q_{p}: X \rightarrow \tilde{X}_{p}$ the quotient map.

Using Randtke's idea(see[8,p.90] or Wong[12,(3.2.1)] and (3.2.7)) we obtain the following interesting results:

Lemma 3.3 Let $\mathfrak{A}$ be an operator ideal on $B$-spaces and $E \in \mathbb{B}$. Then a seminorm $p$ on $E$ is an $\mathfrak{A}$-seminorm if and only if $Q_{p} \in \mathfrak{A}^{i n j}\left(E, \tilde{E}_{p}\right)$.

Proof. By Lemma 3.2, $\mathfrak{A}^{i n j}\left(E, \tilde{E}_{p}\right)=\mathfrak{L}\left(E_{\mathfrak{P}(\mathfrak{A})}, \tilde{E}_{p}\right)$, the result then follows from the following equivalent statements:

$\mathrm{p}$ is an $\mathfrak{A}$-seminorm

$$
\begin{aligned}
& \Leftrightarrow \mathrm{p} \text { is } \mathfrak{P}(\mathfrak{A})(E) \text {-continuous } \\
& \Leftrightarrow Q_{p} \in \mathfrak{L}\left(E_{\mathfrak{P}(\mathfrak{A})}, \tilde{E}_{p}\right) .
\end{aligned}
$$


If $\mathfrak{A}$ is assumed to be injective operator ideal on $\mathfrak{b}$ then the preceding result can be extended to the case of operator ideals on LCS's as shown by the following:

Theorem 3.4 Let $\mathfrak{A}$ be an injective operator ideal on $\mathfrak{b}$ and $X \in \mathfrak{b}$. Then a seminorm $p$ on $X$ is an $\mathfrak{A}$-seminorm if and only if $Q_{p} \in \mathfrak{A}\left(X, X_{p}\right)$.

Proof. Necessity. Let p be an $\mathfrak{A}$-seminorm on X. Then there exists an $\left(Y, \mathfrak{T}_{\text {ori }}(Y)\right) \in \mathfrak{b}$, an $T \in \mathfrak{A}(X, Y)$ and a $\mathfrak{T}_{\text {ori }}(Y)$-continuous seminorm $\mathrm{r}$ on $\mathrm{Y}$ such that

$$
p(x) \leqq r(T x) \quad(\text { for all } x \in X),
$$

so that

$$
K e r T \subseteq K \operatorname{Kerp}=\operatorname{Ker} Q_{p} .
$$

Let $Y_{0}=T(X)$ be equpped with the relative topology, let $J_{Y_{0}}: Y_{0} \rightarrow Y$ be the canonical embedding, and let us define $T_{0}: X \rightarrow Y_{0}$ by setting

$$
T_{0} x=T x \quad(\text { for all } x \in X) .
$$

Then $T_{0} \in \mathfrak{L}(X, Y)$ is surjective and $J_{Y_{0}}$ is a topological injection such that

$$
J_{Y_{0}} T_{0}=T
$$

it then follows from $T \in \mathfrak{A}(X, Y)$ and the injective of $\mathfrak{A}$ that $T_{0} \in \mathfrak{A}\left(X, Y_{0}\right)$.

On the other hand, (2) and (3) show that

$$
\operatorname{Ker} T_{0}=K e r T \subseteq K e r Q_{p},
$$

it then follows from (1) and (4) that there exists an $R \in \mathfrak{L}\left(Y_{0}, X_{p}\right)$ such that $Q_{p}=R T_{0}$, and hence from $T_{0} \in \mathfrak{L}\left(X, Y_{0}\right)$ that $Q_{p} \in \mathfrak{A}\left(X, X_{p}\right)$.

Sufficient. As $Q_{p} \in \mathfrak{A}\left(X, X_{p}\right)$ and $\hat{p}$ is a continuous seminorm on the normed space $X_{p}$ it follows from

$$
p(x)=\hat{p}\left(Q_{p}(x)\right) \quad(\text { for all } x \in X)
$$

that $\mathrm{p}$ is an $\mathfrak{A}$-seminorm.

The preceeding result has some interesting applications, to see this, let us recall the following terminologies:

Let $\mathrm{X}$ be a locally convex space. A seminorm $\mathrm{p}$ on $\mathrm{X}$ is:

(a) precompact (see Randtke $\left[8\right.$, p.90]) if there exists an $\left(\lambda_{n}\right) \in c_{0}$ and an equicontinuous sequence $\left(f_{n}\right)$ in $X^{\prime}$ such that

$$
p(x) \leqq \sup _{n}\left|\lambda_{n} f_{n}(x)\right| \quad(\text { for all } x \in X) ;
$$


(b) prenuclear (see Schaefer $\left[9\right.$, p.177]) if there exists a $\sigma\left(X, X^{\prime}\right)$-closed equicontinuous subset $\mathrm{B}$ of $X^{\prime}$ and a positive Radon measure $\mu$ on B such that

$$
p(x) \leqq \int_{B}|<x, f>| d \mu(f) \quad(\text { for all } x \in X) .
$$

In addition, $\left(X, X_{+}, \mathfrak{I}\right)$ is a locally solid space (for definition, see[12]), $\mathrm{p}$ is a

(c) (PL)-seminorm (see Wong[12, p.126]) if there exists a positive $f \in X^{\prime}$ such that

$$
p(x) \leqq \sup \{g(x):-f \leqq g \leqq f\} \quad \text { (for all } x \in X) .
$$

The class of all precompact (resp. absolutely summing(for definition, see[12, p.121])) operators, denoted by $\mathfrak{L}^{p}\left(\right.$ resp. $\left.\mathfrak{L}^{s}\right)$, is an injective operator ideal on $\mathbb{L}$.

It is shown by Randtke[8, p.90] that a seminorm $\mathrm{p}$ on $\mathrm{X}$ is precompact if and only if $Q_{p} \in \mathfrak{L}^{S}\left(X, X_{p}\right)$. On the other hand, it is known(see [12,(3.2.1)]) that a seminorm $\mathrm{p}$ on a locally solid $\operatorname{space}\left(X, X_{+}, \mathfrak{I}\right)$ is a $(\mathrm{PL})$-seminorm if and only if $Q_{p} \in \mathfrak{L}^{l}\left(X, X_{p}\right)$ (coneabsolutely summing). Therefore, precompact[resp. prenuclear, (PL)-seminorm] seminorms are $\mathfrak{L}^{p}$-seminorms(resp. $\mathfrak{L}^{s}$-seminorms, $\mathfrak{L}^{l}$-seminorms), in other words, Theorem 3.4 is a generalization of a result of Randtke[8,(2.5)] and of results of Wong[12,(3.2.7) and (3.2.1)].

We shall now turn our attention to matters related to the question (Q2), namely: what kind of compactible locally convex topologies $\mathfrak{P}$ will ensure that the setting:

$$
\mathfrak{A}[\mathfrak{P}](X, Y)=\mathfrak{L}\left(X_{\mathfrak{P}}, Y\right) \quad(\text { for all } X, Y \in \mathfrak{b})
$$

defines an operator ideal $\mathfrak{A}[\mathfrak{P}]$ on $\mathfrak{b}$ ? It is clear that $\mathfrak{F} \subseteq \mathfrak{A}[\mathfrak{P}]$ and that $\mathfrak{A}[\mathfrak{P}](X, Y)$ is a vector space. The following result gives a criterion a for $\mathfrak{A}[\mathfrak{P}]$ to satisfy the ideal property $\left(O I_{2}\right)$.

Lemma 3.5 Let $\mathfrak{g}=\{\mathfrak{g}(X): X \in \mathfrak{b}$ be a family of Hausdorff locally convex topologies on all members in $\mathfrak{b}$ and

$$
X_{\mathfrak{g}}=(X, \mathfrak{g}(X)) \quad(\text { for all } X \in \mathfrak{b})
$$

Then the following statements are equivalent.

(a) Suppose that $G, X, Y, Z \in \mathfrak{b}$ and that

$$
R \in \mathfrak{L}(Y, Z), \quad T \in \mathfrak{L}\left(X_{\mathfrak{g}}, Y\right) \quad \text { and } \quad S \in \mathfrak{L}(G, X) .
$$


Then $R T S \in \mathfrak{L}\left(G_{\mathfrak{g}}, Z\right)$.

(b) $\mathfrak{L}(X, Y) \subseteq \mathfrak{L}\left(X_{\mathfrak{g}}, Y_{\mathfrak{g}}\right)($ for all $X, Y \in \mathfrak{b})$.

Proof. The implication (b) $\Rightarrow$ (a) is obvious. To prove that (a) implies (b), let $T \in \mathfrak{L}(X, Y)$ and $\mathrm{q}$ a $\mathfrak{g}(Y)$-continuous seminorm on $\mathrm{Y}$. Then the quotient map $Q_{q}$ : $Y_{\mathfrak{g}} \rightarrow Y_{q}=\left(Y / q^{-1}(0), \hat{q}\right)$ is continuous, hence $Q_{q} T \in \mathfrak{L}\left(X_{\mathfrak{g}}, Y_{q}\right)$ by (a) and $\left.T \in \mathfrak{L}(X, Y)\right]$, and thus there is a $\mathfrak{g}(X)$-continuous seminorm $\mathrm{p}$ on $\mathrm{X}$ such that

$$
\hat{q}\left(Q_{q}(T x) \leqq p(x)\right) \quad(\text { for all } x \in X) .
$$

We conclude from $\hat{q}\left(Q_{q}(T x)\right)=q(T x)$ that $T \in \mathfrak{L}\left(X_{\mathfrak{g}}, Y_{\mathfrak{g}}\right)$.

Definition 3.6 (Stephani[11], Franco/Piñeiro[3]). For any $X \in \mathfrak{b}$, let $\mathfrak{g}(X)$ be a locally convex topology on $\mathrm{X}$ (which may be different from $\left.\mathfrak{T}_{\text {ori }}(X)\right)$. The collection

$$
\mathfrak{g}=\{\mathfrak{g}(X): X \in \mathfrak{b}\}
$$

is called a generating topology on $\mathfrak{b}$ if it satisfies the following two conditions:

$\left(G T_{1}\right) \mathfrak{g}(X) \leqq \mathfrak{T}_{\text {ori }}(X)$ for any $X \in \mathfrak{b}$

$\left(G T_{2}\right) \mathfrak{L}(X, Y) \subseteq \mathfrak{L}\left(X_{\mathfrak{g}}, Y_{\mathfrak{g}}\right)($ for any $X, Y \in \mathfrak{b})$, where $X_{\mathfrak{g}}=(X, \mathfrak{g}(X))$

Remark. As $\mathbb{K} \in \mathfrak{b}$ and $\mathfrak{g}(\mathbb{K})=\|\cdot\|_{\mathbb{K}}$-topology, it follows that $\mathfrak{g}(X)$ is consistent with $<X, X^{\prime}>$ for any $X \in \mathfrak{b}$. On the other hand, if $\mathfrak{b}=\mathbb{B}$ then one can show (see [11]) that

$$
\mathfrak{L}(E, F)=\mathfrak{L}\left(E_{\mathfrak{g}}, F_{\mathfrak{g}}\right) \quad \text { for all } E, F \in \mathbb{B} .
$$

However the preceeding equality is, in general, not true unless $\mathfrak{b}$ is a subclass of all Mackey spaces. Indeed, consider an infinite-dimensional normed space E, the identity map $i d_{E}$ on $E$ is clearly weak-weak continuous but not weak-norm continuous. This says that

$$
\mathfrak{L}\left(E_{\sigma}, E\right) \neq \mathfrak{L}\left(E_{\sigma}, E_{\sigma}\right) .
$$

However, it is easily seen that the system $\mathfrak{g}_{\sigma}$ of weak topologies is a generating topology on locally convex spaces.

Let $\mathfrak{g}$ be a generating topology on $\mathfrak{b}$. Then Lemma 3.5 and Definition 3.6 show that the class $\mathfrak{O}[\mathfrak{g}]$ of operators, defined by

$$
\left.\mathfrak{O}[\mathfrak{g}](X, Y)=\mathfrak{L}\left(X_{\mathfrak{g}}, Y\right) \quad \text { (for all } X, Y \in \mathfrak{b}\right),
$$

is an operator ideal on $\mathfrak{b}$; moreover, it is injective as shown by the following: 
Lemma 3.7 Let $\mathfrak{g}$ be a generating topology on $\mathfrak{b}$. Then the class $\mathfrak{O}[\mathfrak{g}]$, defined by

$$
\mathfrak{O}[\mathfrak{g}](X, Y)=\mathfrak{L}\left(X_{\mathfrak{g}}, Y\right) \quad(\text { for all } X, Y \in \mathfrak{b}),
$$

is an injective operator ideal on $\mathfrak{b} ;$ it is called the injective operator ideal on $\mathfrak{b}$ associated with $\mathfrak{g}$.

Proof. Suppose that $T \in \mathfrak{L}(X, Y), Z \in \mathfrak{b}$ and that $J: Y \rightarrow Z$ is a topological injection such that $J T \in \mathfrak{O}[\mathfrak{g}](X, Z)$. It is required to show that $T \in \mathfrak{O}[\mathfrak{g}](X, Y)\left(=\mathfrak{L}\left(X_{\mathfrak{g}}, Y\right)\right)$. In fact, let $\mathrm{U}$ be a 0 -neighbourhood in $\mathrm{Y}$, and let $\mathrm{W}$ be a 0 -neighbourhood in $\mathrm{Z}$ such that $W \cap J(Y) \subseteq J(U)$. It then follows from the injectivity of $\mathrm{J}$ that

$$
J^{-1}(W) \subseteq U .
$$

As $J T \in \mathfrak{L}\left(X_{\mathfrak{g}}, Z\right)(=\mathfrak{O}[\mathfrak{g}](X, Y))$, it follows that there exists a $\mathfrak{g}(X)$-neighbourhood $\mathrm{V}$ of 0 in $\mathrm{X}$ such that

$$
V \subseteq(J T)^{-1}(W)=T^{-1}\left(J^{-1}(W)\right),
$$

and hence from (1) that $V \subseteq T^{-1}(U)$, thus $T \in \mathfrak{L}\left(X_{\mathfrak{g}}, Y\right)$.

The preceeding result was proved by Stephan[11,p.201] in the special case $\mathfrak{b}=\mathbb{B}$.

The following result demonstrates some relationship between ideal-topologies and generating topologies.

Theorem 3.8 (a)Given an operator ideal $\mathfrak{A}$ on $\mathfrak{b}$. Then the $\mathfrak{A}$-topology $\mathfrak{P}(\mathfrak{A})$ on $\mathfrak{b}$ is a generating topology on $\mathfrak{b}$. If, in addition, $\mathfrak{b}=\mathbb{B}$, then the injective operator ideal $\mathfrak{O}[\mathfrak{P}(\mathfrak{A})]$ associated with $\mathfrak{P}(\mathfrak{A})$ is the injective hull of $\mathfrak{A}$, that is

$$
\mathfrak{O}[\mathfrak{P}(\mathfrak{A})]=\mathfrak{A}^{i n j} .
$$

Unfortunately the preceeding equality is no longer true for $\mathfrak{b} \neq \mathbb{B}$.

(b) Given a generating topology $\mathfrak{g}$ on $\mathfrak{b}$, let $\mathfrak{O}[\mathfrak{g}]$ be the injective operator ideal on $\mathfrak{b}$ associated with $\mathfrak{g}$. Then $\mathfrak{g}$ is the $\mathfrak{O}[\mathfrak{g}]$-topology on $\mathfrak{b}$, that is

$$
\mathfrak{g}=\mathfrak{P}(\mathfrak{O}[\mathfrak{g}]) ;
$$

consequently, every generating topology on $\mathfrak{b}$ is an ideal-topology on $\mathfrak{b}$.

Proof. (a)For any $X \in \mathfrak{b}$, it is known from Definition 3.1 that $\mathfrak{P}(\mathfrak{A})(X)$ is consistent with $<X, X^{\prime}>$. To check that

$$
\left.\mathfrak{L}(X, Y) \subseteq \mathfrak{L}\left(X_{\mathfrak{P}(\mathfrak{A})}, Y_{\mathfrak{P}(\mathfrak{A})}\right) \quad \text { (for all } X, Y \in \mathfrak{b}\right),
$$

we first notice that the $\mathfrak{P}(\mathfrak{A})(X)$-topology on $\mathrm{X}$ is generated by the following family of seminorms: 


$$
\begin{aligned}
\mathfrak{l}(\mathfrak{A})(X)=\left\{q_{Y} \circ T: T \in \mathfrak{A}(X, Y),\right. \\
\left.q_{Y} \text { is } \mathfrak{T}_{\text {ori }}(Y) \text {-continuous seminorms, } Y \in \mathfrak{b}\right\} .
\end{aligned}
$$

Now, let $T \in \mathfrak{L}(X, Y)$ and $\mathrm{r}$ an $\mathfrak{P}(\mathfrak{A})$-continuous seminorm on $Y$. Then there exists an $Z \in \mathfrak{b}$, an $S \in \mathfrak{A}(Y, Z)$ and a $\mathfrak{T}_{\text {ori }}(Z)$-continuous seminorm $q_{Z}$ on $\mathrm{Z}$ such that

$$
r(y) \leqq q_{Z}(S y) \quad \text { for all } y \in Y
$$

in particular,

$$
r(T x) \leqq q_{Z}(S T x) \quad \text { for all } x \in X .
$$

As $S T \in \mathfrak{A}(X, Z)$, it follows that $p=q_{Z} \circ S \circ T$ is an $\mathfrak{A}$-seminorm on X such that

$$
r(T x) \leqq p(x) \quad \text { for all } x \in X,
$$

and hence that $T \in \mathfrak{L}\left(X_{\mathfrak{P}(\mathfrak{A})}, Y_{\mathfrak{P}(\mathfrak{A})}\right)$ as required.

Suppose now that $\mathfrak{A}$ is an operator ideal on B-spaces, and that $E, F \in \mathbb{B}$. Then Lemma3.2 and 3.7 show that

$$
\mathfrak{A}^{i n j}(E, F)=\mathfrak{L}\left(E_{\mathfrak{P}(\mathfrak{A})}, F\right)=\mathfrak{O}[\mathfrak{P}(\mathfrak{A})](E, F) .
$$

Equality(3.8.1)is, in general, not true for operator ideals on LCS's as shown by the following example: consider the operator ideal $\mathfrak{F}$ on LCS's of continuous finite operators. It is easily seen that $\mathfrak{F}$ is injective on $\mathbb{L}$ and that

$$
\mathfrak{P}(\mathfrak{F})=\mathfrak{g}_{\sigma}=\left\{\sigma\left(X, X^{\prime}\right): X \in \mathbb{L}\right\} .
$$

Now for an finite-dimensional normed space $X$, it then follows from Lemma3.7 that

$$
i d_{X} \in \mathfrak{L}\left(X_{\sigma}, X_{\sigma}\right)=\mathfrak{O}[\mathfrak{P}(\mathfrak{F})]\left(X_{\sigma}, X_{\sigma}\right) ;
$$

but $i d_{X} \nsubseteq \mathfrak{F}\left(X, X_{\sigma}\right)$ unless $\operatorname{dim} X<\infty$. This shows that $\mathfrak{F} \varsubsetneqq \mathfrak{O}[\mathfrak{P}(\mathfrak{F})]$.

(b) Let $\mathfrak{g}$ be a generating topology on $\mathfrak{b}$. On each $X \in \mathfrak{b}$, the ideal-topology $\mathfrak{P}(\mathfrak{O}[\mathfrak{g}])(X)$ on $\mathrm{X}$ is the coarsest topology on $\mathrm{X}$ for which all operators in $\mathfrak{O}[\mathfrak{g}](X, Y)$ are $\mathfrak{P}(\mathfrak{O}[\mathfrak{g}])(X)$-continuous (whenever $Y \in \mathfrak{b})$. As $\mathfrak{O}[\mathfrak{g}](X, Y)=\mathfrak{L}\left(X_{\mathfrak{g}}, Y\right)$, we conclude that

$$
\mathfrak{P}(\mathfrak{O}[\mathfrak{g}])(X) \leqq \mathfrak{g}(X) \quad(\text { for all } X \in \mathfrak{b}) .
$$

Conversely, let p be a $\mathfrak{g}(X)$-continuous seminorm on X. Then $Q_{p} \in \mathfrak{L}\left(X_{\mathfrak{g}}, X_{p}\right)\left(=\mathfrak{O}[\mathfrak{g}]\left(X, X_{p}\right)\right)$. As the operator ideal $\mathfrak{O}[\mathfrak{g}]$ is injective(see Lemma 3.7), it follows from Theorem 3.4 that $\mathrm{p}$ is an $\mathfrak{O}[\mathfrak{g}]$-seminorm on $\mathrm{X}$ or equivalently, p is $\mathfrak{P}(\mathfrak{O}[\mathfrak{g}])(X)$-continuous; consequently, $\mathfrak{g}(X) \leqq \mathfrak{P}(\mathfrak{O}[\mathfrak{g}])(X)$.

Following Franco and Piñeiro [3], a subclass $\mathbb{P}$ of the class $\mathfrak{T}_{\text {ori }}$ of all the continuous seminorms on $\mathbb{L}$ is called a seminorm ideal if all the components $\mathbb{P}(X):=\mathbb{P} \cap \mathfrak{T}_{\text {ori }}(X)$ 
with $X \in \mathbb{L}$ satisfy the following conditions:

$\left(P_{1}\right)$ : for all $x^{\prime} \in X^{\prime}$, there is an $p \in \mathbb{P}(X)$ and an $\alpha>0$ such that

$$
\left|<x, x^{\prime}>\right| \leqq \alpha p(x) \quad \forall x \in X
$$

$\left(P_{2}\right)$ : for any $p_{1}$ and $p_{2} \in \mathbb{P}(X)$, there is an $p \in \mathbb{P}(X)$ such that

$$
p_{i} \leqq p \quad(\mathrm{i}=1,2) ;
$$

$\left(P_{3}\right)$ : for all $q \in \mathbb{P}(X), Y \in \mathbb{L}$ and $T \in \mathfrak{L}(X, Y)$, there is an $p \in \mathbb{P}(X)$ and an $\alpha>0$ such that

$$
q(T(x)) \leqq \alpha p(x) \quad \forall x \in X
$$

$\left(P_{4}\right)$ : if $p \leqq q$ with $q \in \mathbb{P}(X)$ (where $\mathrm{p}$ is a seminorm on $\mathrm{X}$ ), then $p \in \mathbb{P}(X)$.

One can easily obtain the Banach spaces version of the above definition.

We summarize the above discussion by the following:

Theorem 3.9 Let $\mathfrak{b}$ be either $\mathbb{B}$ or $\mathbb{L}$, and let

$$
\mathfrak{g}=\{\mathfrak{g}(X): X \in \mathfrak{b}
$$

be a family of locally convex topologies on $\mathfrak{b}$. Consider the following statements:

(a) $\mathfrak{L}(X, Y)=\mathfrak{L}\left(X_{\mathfrak{g}}, Y_{\mathfrak{g}}\right) \quad$ for all $X, Y \in b$.

(b) $\mathfrak{g}$ is an $\mathfrak{A}$-topology on $\mathfrak{b}$ for some operator ideal $\mathfrak{A}$ on $\mathfrak{b}$.

(c) $\mathfrak{g}$ is a generating topology on $\mathfrak{b}$.

(d) The class defined by

$$
\mathfrak{O}[\mathfrak{g}](X, Y)=\mathfrak{L}\left(X_{\mathfrak{g}}, Y\right) \quad \text { for all } X, Y \in \mathfrak{b},
$$

is an injective operator ideal on $\mathfrak{b}$. 
(e) The family of all $\mathfrak{g}$-continuous seminorms constitutes a seminorm ideal on $\mathfrak{b}$. If $\mathfrak{b}=\mathbb{L}$ then we have

$$
(a) \Rightarrow(b) \Leftrightarrow(c) \Leftrightarrow(d) \Leftrightarrow(e) .
$$

If $\mathfrak{b}=\mathbb{B}$ then we have

$$
(a) \Leftrightarrow(b) \Leftrightarrow(c) \Leftrightarrow(d) \Leftrightarrow(e) .
$$

Proof. Then equivalences $(b) \Leftrightarrow(c) \Leftrightarrow(d) \Leftrightarrow(e)$ are easy to verify $(a) \Rightarrow(c)$ is obvious. For $\mathfrak{b}=\mathbb{B}$, it is known from Schaefer $[9$, p.158] that every continuous operator from a $\mathbb{L} \mathbb{C}$ into another is also continuous for the Mackey topologies on these two spaces. Since Banach spaces are Mackey, we have $\mathfrak{L}\left(E_{\mathfrak{g}}, F_{\mathfrak{g}}\right) \subseteq \mathfrak{L}(E, F)$ (where $\left.E, F \in \mathbb{B}\right)$. Therefore, the implication $(c) \Leftrightarrow(a)$ now comes from $\left(G T_{2}\right)$.

The equivalence $(c) \Leftrightarrow(e)$ is mentioned by Franco and Piñeiro [3]. Also the equivalence of (a) and (c) is due to Stephani[11]; but we give here a different and quite easier proof.

It is remarkable that the preceeding result contains an interesting fact that every system $\mathfrak{g}$ of locally convex topologies on the category $\mathfrak{b}=\mathbb{B}$ (or the class of all Mackey spaces) satisfying the condition

$$
\mathfrak{L}\left(X_{\mathfrak{g}}, Y_{\mathfrak{g}}\right)=\mathfrak{L}(X, Y) \quad \text { for all } X, Y \in \mathfrak{b}
$$

is nothing but an $\mathfrak{A}$-topology. In other words, to study different kinds of operator ideals on $\mathfrak{b}$ it is to study such the systems of locally convex topologies, and vice versa. Here, we give out some interesting examples.

Example 3.10 3.10. Denote respectively by $\mathfrak{g}_{p c}, \mathfrak{g}_{q p v}, \mathfrak{g}_{p v}$, and $\mathfrak{g}_{s v}$ the generating systems of precompact topologies, quasi-p-nuclear topologies, prenuclear topologies and strongly nuclear topologies induced by the corresponding ideals of precompact operators, quasi-p-nuclear operators, absolutely summing operators and strongly nuclear operators (ideal definitions of these generating topologies can be found in Wong[13,p.14,161,146]). With these notations, we have for all $E$ and $F \in \mathbb{B}$ that

$$
\begin{aligned}
\mathfrak{L}(X, & Y)=\mathfrak{L}\left(X_{\mathfrak{g}_{p c}}, Y_{\mathfrak{g}_{p c}}\right) \\
& =\mathfrak{L}\left(X_{\mathfrak{g}_{q p v}}, Y_{\mathfrak{g}_{q p v}}\right) \\
& =\mathfrak{L}\left(X_{\mathfrak{g}_{p v}}, Y_{\mathfrak{g}_{p v}}\right) \\
& =\ldots
\end{aligned}
$$




\section{Ideal-Bornologies and Generating Bornolobies}

Using the notion of bornologies, this section is devoted to a study of the dual concepts of ideal-topologies and generating topologies.

Throughout this paper, $\mathfrak{U}_{\text {von }}(X)$ denotes the von Neumann bornology on $X \in \mathbb{L}$ and by referring $\mathrm{X}$ we always mean the bornological space $\left(X, \mathfrak{U}_{\text {von }}(X)\right)$.

It is clear the operator ideal $\mathfrak{F}$ on $\mathbb{L}$ is surjective, moreover, we have, for any normed space $\mathrm{E}$ and $Y \in \mathbb{L}$, that

$$
\mathfrak{F}(E, Y)=L^{x}\left(E, Y^{\mathfrak{U}_{f}}\right) \cap \mathfrak{L}(E, Y),
$$

where $Y^{\mathfrak{U}_{f}}=\left(Y, \mathfrak{U}_{f}(Y)\right)$ and $\mathfrak{U}_{f}(Y)$ is the finite-dimensional bornology on $\mathrm{Y}$.

(4a)suggests the following question which is dual to (Q1) of 3.

$(Q 1)^{*}$ Let $\mathfrak{A}$ be a given operator ideal on $\mathfrak{b}$. For any $X \in \mathfrak{b}$, does there exist a convex bornology $\mathfrak{B}$ such that

$$
\mathfrak{A}(X, Y)=L^{x}\left(X, Y^{\mathfrak{B}}\right) \cap \mathfrak{L}(X, Y) \quad(\text { for all } Y \in \mathfrak{b}) ?
$$

Conversely, it is natural to ask the following question [it is a dual version of (Q2) in $3]$ :

$(Q 2)^{*}$ What kind of convex bornologies $\mathfrak{B}$ with $\mathfrak{U}_{\text {von }}(X) \leqq \mathfrak{B}(X) \leqq \mathfrak{U}_{f}(X)$ (for all $X \in \mathfrak{b})$ will ensure that the setting

$$
\mathfrak{O}[\mathfrak{B}](X, Y)=L^{x}\left(X, Y^{\mathfrak{B}}\right) \cap \mathfrak{L}(X, Y) \quad(\text { for all } X, Y \in \mathfrak{b})
$$

defines an operator ideal $\mathfrak{O}[\mathfrak{B}]$ on $\mathfrak{b}$ ?

In order to answer the question $(Q 1)^{*}$, we require the following terminology which is dual to (3.1).

Definition 4.1 Let $\mathfrak{A}$ be an operator ideal on $\mathfrak{b}$. For a given $Y \in \mathfrak{b}$, let $\mathfrak{U}(\mathfrak{A})(Y)$ be the final convex bornology on $\mathrm{Y}$ with respect to the family

$$
\left\{\left(X, \mathfrak{U}_{\text {von }}(X), S\right): S \in \mathfrak{A}(X, Y) \text { and } X \in \mathfrak{b}\right\} .
$$

Then the collection, defined by

$$
\mathfrak{U}(\mathfrak{A})=\{\mathfrak{U}(\mathfrak{A})(Y): Y \in \mathfrak{b}\},
$$

is called the $\mathfrak{A}$-bornology (or ideal-bornology) on $\mathfrak{b}$. On each $Y \in \mathfrak{b}$, members in $\mathfrak{U}(\mathfrak{A})(Y)$ are said to be $\mathfrak{A}$-bounded (or ideal-bounded) in $\mathfrak{b}$; also we write

$$
Y^{\mathfrak{U}(\mathfrak{A})}=(Y, \mathfrak{U}(\mathfrak{A})(Y)) \quad(\text { for all } Y \in \mathfrak{b}) .
$$

By the definition of final convex bornologies, it is easily seen that

$$
\mathfrak{U}_{f}(Y) \subseteq \mathfrak{U}(\mathfrak{A})(Y) \subseteq \mathfrak{U}_{\text {von }}(Y) \quad(\text { for all } Y \in \mathfrak{b})
$$

and that

$$
\mathfrak{A}(X, Y) \subseteq L^{x}\left(X, Y^{\mathfrak{U}(\mathfrak{A})}\right) \cap \mathfrak{L}(X, Y) \quad(\text { for all } X, Y \in \mathfrak{b}) .
$$

On the other hand, $\mathfrak{U}(\mathfrak{A})(Y)$ is the intersection of all convex bornologies containing 


$$
\mathfrak{U}_{0}(\mathfrak{A})(Y)=\cup\left\{S\left(\mathfrak{U}_{\text {von }}(X)\right): S \in \mathfrak{A}(X, Y), X \in \mathfrak{b}\right\},
$$

where $S\left(\mathfrak{U}_{\text {von }}(X)\right)=\left\{S(A): A \in \mathfrak{U}_{\text {von }}(X)\right\}$. Moreover, $\mathfrak{U}_{0}(\mathfrak{A})(Y)$ is a base for $\mathfrak{U}(\mathfrak{A})(Y)$ as shown by the following:

Lemma 4.2 Let $\mathfrak{A}$ be an operator ideal on $\mathfrak{b}$. For any $Y \in \mathfrak{b}$, the family $\mathfrak{U}_{0}(\mathfrak{A})(Y)$ defined by (4b), is a base for the ideal-bornology $\mathfrak{U}(\mathfrak{A})(Y)$ on $Y$. Consequently, a subset $B$ of $Y$ is $\mathfrak{A}$-bounded if and only if there exists an $Z \in \mathfrak{b}$, an $S \in \mathfrak{A}(Z, Y)$ and an $A \in \mathfrak{U}_{\text {von }}(Z)$ such that

$$
B \subseteq S(A)
$$

Proof. To check that $\mathfrak{U}_{0}(\mathfrak{A})(Y)$ is a base for a convex bornology, it has to show, in view of the linearity of each $S \in \mathfrak{A}(X, Y)$ and $X \in \mathfrak{b}$, that $\mathfrak{U}_{0}(\mathfrak{A})(Y)$ satisfies the following two properties:

(i) $Y=\cup \mathfrak{U}_{0}(\mathfrak{A})(Y)$;

(ii) if $S_{i} \in \mathfrak{A}\left(X_{i}, Y\right)$ and $A_{i} \in \mathfrak{U}_{\text {von }}\left(X_{i}\right)(\mathrm{i}=1,2)$, then there exists an $X \in \mathfrak{b}$, an $S \in$ $\mathfrak{A}(X, Y)$ and $A \in \mathfrak{U}_{\text {von }}(X)$ such that $S_{1}\left(A_{1}\right)+S_{2}\left(A_{2}\right) \subseteq S(A)$.

In fact, for any $y \in Y$, let $Z=\{\lambda y: y \in \mathbb{K}\}$ be equipped with the relative topology and $T \in J_{Z}$ (the canonical injection). Then

$$
T \in \mathfrak{F}(Z, Y) \subseteq \mathfrak{A}(Z, Y) \text { and } B=\{y\} \in \mathfrak{U}_{\text {von }}(Z)
$$

are such that $y \in T(B) \in \cup \mathfrak{U}_{0}(\mathfrak{A})$, so that (i) holds. To prove (ii), we first notice that the map $S_{1} \times S_{2}$, defined by

$$
S_{1} \times S_{2}: X_{1} \times X_{2} \rightarrow Y \times Y:\left(x_{1}, x_{2}\right) \rightarrow\left(S_{1} x_{1}, S_{2} x_{2}\right),
$$

belongs to $\mathfrak{A}\left(X_{1} \times X_{2}, Y_{1} \times Y_{2}\right)$ since $S_{i} \in \mathfrak{A}\left(X_{i}, Y\right)$ and

$$
S_{1} \times S_{2}=J_{Y}^{(1)} S_{1} \Pi_{X_{1}}+J_{Y}^{(2)} S_{2} \Pi_{X_{2}},
$$

where $\Pi_{X_{i}}: X_{1} \times X_{2} \rightarrow X_{i}$ is the ith projection and

$$
J_{Y}^{(1)} y=(y, 0) \text { and } J_{Y}^{(2)} y=(0, y) \quad(\text { for all } y \in Y) .
$$

On the other hand, let us define

$$
\begin{aligned}
& \Pi_{Y}^{(1)}: Y \times Y \rightarrow Y:\left(y_{1}, y_{2}\right) \mapsto y_{1} \\
& \Pi_{Y}^{(2)}: Y \times Y \rightarrow Y:\left(y_{1}, y_{2}\right) \mapsto y_{2},
\end{aligned}
$$

whenever $\left(y_{1}, y_{2}\right) \in Y \times Y$. Then 


$$
S=\left(\Pi_{Y}^{(1)}+\Pi_{Y}^{(2)}\right)\left(S_{1} \times S_{2}\right) \in \mathfrak{A}\left(X_{1} \times X_{2}, Y\right)
$$

and $A=A_{1} \times A_{2} \in \mathfrak{U}_{\text {von }}\left(X_{1} \times X_{2}\right)$ are such that

$$
\begin{aligned}
S(A) & =S\left(A_{1} \times A_{2}\right)=\left(\Pi_{Y}^{(1)}+\Pi_{Y}^{(2)}\right) S_{1} \times S_{2}\left(A_{1} \times A_{2}\right) \\
& =S_{1}\left(A_{1}\right)+S_{2}\left(A_{2}\right) \in \mathfrak{U}_{0}(\mathfrak{A})(Y)
\end{aligned}
$$

(since $X=X_{1} \times X_{2} \in \mathfrak{b}$ ). This proves our assertion (ii).

Finally, let $\mathfrak{B}$ be the convex bornology on $Y$ generated by $\mathfrak{U}_{0}(\mathfrak{A})(Y)$, that is

$\mathfrak{B}=\left\{B \subseteq Y: B \subseteq D\right.$ for some $\left.D \in \mathfrak{U}_{0}(\mathfrak{A})(Y)\right\}$.

Then $\mathfrak{U}(\mathfrak{A})(Y) \subseteq \mathfrak{B}$ [by the definition of $\mathfrak{U}(\mathfrak{A})(Y)$ ]. Conversely, since $\mathfrak{U}(\mathfrak{A})(Y)$ is a convex bornology containing $\mathfrak{U}_{0}(\mathfrak{A})(Y)$, it follows from the definition of $\mathfrak{B}$ that $\mathfrak{B} \subseteq \mathfrak{U}(\mathfrak{A})(Y)$. Therefore the family $\mathfrak{U}_{0}(\mathfrak{A})(Y)$ is a base for $\mathfrak{U}(\mathfrak{A})(Y)$. Consequently (4.2.1) holds (by the definition of $\mathfrak{A}$-bounded sets and bases).

Base on Lemma 4.2, we are now able to give dual versions of all results in Sect.3.

Lemma 4.3 Let $\mathfrak{A}$ be an operator ideal on $\mathbb{B}$. Then

$$
L^{x}\left(E, F^{\mathfrak{U}(\mathfrak{A})}\right)=\mathfrak{A}^{\text {sur }}(E, F) \quad(\text { for all } E, F \in \mathbb{B}) .
$$

Moreover, (4.3.1) is no longer true for an operator ideal $\mathfrak{A}$ on $\mathfrak{b} \neq \mathbb{B}$.

Proof.(4.3.1) follows immediately from Lemma 2.1(b) and (4.2.1) of Lemma 4.2.

Finally, $\mathfrak{L}$ is an operator ideal on $\mathbb{L}$ with

$$
\mathfrak{U}(\mathfrak{L})(X)=\mathfrak{U}_{\text {von }}(X) \quad \text { for all } \quad X \in \mathbb{L}
$$

(i.e., the system of all Von Neumann bornologies is an ideal-bornology), hence

$$
L^{x}\left(X, Y^{\mathfrak{U}(\mathfrak{L})}\right)=L^{x}(X, Y) \supsetneqq \mathfrak{L}(X, Y)
$$

in general (unless $\mathrm{X}$ is a bornological locally convex space).

In order to give a characterization of $\mathfrak{A}$-bounded sets, we require the following notations. For any absolutely convex subset B of a vector space $\mathrm{Y}$, let $Y(B)=\bigcup_{n \geq 1} n B$ (the vector subspace of $\mathrm{Y}$ generated by $\mathrm{B}$ ), let $r_{B}$ be the gauge of $\mathrm{B}$ defined on $Y(B)$, and let

$$
J_{B}: Y(B) \rightarrow Y
$$

be the canonical embedding. Then $r_{B}$ is a seminorm on $Y(B)$ such that

$$
\left\{y \in Y(B): r_{B}<1\right\} \subseteq B \leqq\left\{y \in Y(B): r_{B} \leqq 1\right\} .
$$

Moreover, if $\mathrm{Y}$ is a locally convex space and $\mathrm{B}$ is bounded in $\mathrm{Y}$, then $r_{B}$ is a norm on $Y(B)$ and $J_{B}:\left(Y(B), r_{B}\right) \rightarrow Y$ is continuous. 
Lemma 4.4 Let $\mathfrak{A}$ be an operator ideal on $B$-spaces and $F \in \mathbb{B}$. Then a bounded set $B$ in $F$ is $\mathfrak{A}$-bounded if and only if $J_{B_{0}} \in \mathfrak{A}^{\text {sur }}\left(F\left(B_{0}\right), F\right)$, where $B_{0}=\Gamma_{\sigma}(B)$ is the $\sigma$-disked hull of $B$.

Proof. By Lemma 4.3, $\mathfrak{A}^{\text {sur }}\left(F\left(B_{0}\right), F\right)=L^{x}\left(F\left(B_{0}\right), F^{\mathfrak{U}(\mathfrak{a})}\right)$, the result then follows from the following equivalence statements:

$$
\begin{array}{cc} 
& J_{B_{0}} \in \mathfrak{A}^{\text {sur }}\left(F\left(B_{0}\right), F\right) \\
\Leftrightarrow & J_{B_{0}}\left(U_{F\left(B_{0}\right)}\right) \in \mathfrak{U}(\mathfrak{A})(F) \\
\Leftrightarrow & B_{0} \text { is } \mathfrak{A} \text {-bounded }\left(\text { since } B_{0} \subseteq U_{F\left(B_{0}\right)} \subseteq 2 B_{0}\right) .
\end{array}
$$

If the operator ideal $\mathfrak{A}$ on $\mathfrak{b}$ is assumed to be surjective, then the preceeding result can be extended to the case of operator ideals on LCS's as shown by the following result which is dual version of Theorem 3.4.

Theorem 4.5 Let $\mathfrak{A}$ be a surjective operator on $\mathfrak{b}$ and $Y \in \mathfrak{b}$. Then a subset $B$ of $Y$ is $\mathfrak{A}$-bounded if and only if the canonical embedding

$$
J_{\Gamma B}:\left(Y(\Gamma B), r_{\Gamma B}\right) \rightarrow Y
$$

belongs to $\mathfrak{A}(Y(\Gamma B), Y)$.

Proof. The sufficiency is trivial by making use of (4.2.1) of Lemma 4.2. To prove the necessity, let B be a absolutely convex. Then there exists an $X \in \mathfrak{b}$, an $T \in \mathfrak{A}(X, Y)$ and $W_{0}=\Gamma W_{0} \in \mathfrak{U}_{\text {von }}(X)$ such that $B \subseteq T W_{0}$, hence the set $\mathrm{W}$, defined by

$$
W=\left(T^{-1} B\right) \cap W_{0},
$$

is an absolutely convex bounded subset of $\mathrm{X}$ such that $T W=B=J_{B}(B)$, so that we have the following commutative diagram:

It then follows from the injectivity of $J_{B}^{T}$ and $\operatorname{Im} J_{B}^{Y}=I m T J_{W}$ that there exists a surjective map $Q: X(W) \rightarrow Y(B)$ such that

$$
T J_{W}=J_{B}^{Y} Q \text {. }
$$

It is easily seen that $\mathrm{Q}$ is linear and

$$
Q(W)=J_{B}^{Y}(Q(W))=T J_{W}(W)=T(W)=B,
$$

hence $\mathrm{Q}$ is open [since $Q\left(U_{X(W)}\right) \supseteqq Q(W)=B$ ] and continuous [since $W \subseteq Q^{-1}(B) \subseteq$ $\left.Q^{-1}\left(U_{Y(B)}\right)\right]$. It then follows from $J_{B}^{Y} Q=T J_{W}$ with $T \in \mathfrak{A}(X, Y)$ that $J_{B} Q \in$ $\mathfrak{A}(X(W), Y)$, and hence from the surjectivity of $\mathfrak{A}$ that $J_{B} \in \mathfrak{A}(Y(B), Y)$ (since $\mathrm{Q}$ is open).

To answer the question $(Q 2)^{*}$, we first verify the following result which is a dual 
version of Lemma 3.5.

Lemma 4.6 Let $\mathfrak{B}=\{\mathfrak{B}(Y): Y \in \mathfrak{b}$ be a family of separated convex bornologies on all members in $\mathfrak{b}$ with $\mathfrak{B}(Y) \subseteq \mathfrak{U}_{\text {von }}(Y)$ and

$$
Y^{\mathfrak{B}}=(Y, \mathfrak{B}(Y)) \quad(\text { for all } y \in \mathfrak{b}) .
$$

Then the following two statements are equivalent.

(a) Suppose that $G, X, Y, Z \in \mathfrak{b}$ and that

$$
R \in \mathfrak{L}(Y, Z), T \in L^{x}\left(X, Y_{\mathfrak{B}}\right) \cap \mathfrak{L}(X, Y) \quad \text { and } \quad S \in \mathfrak{L}(G, X) .
$$

Then $R T S \in L^{x}\left(G, Z^{\mathfrak{B}}\right)$.

(b) $\mathfrak{L}(X, Y) \subseteq L^{x}\left(X^{\mathfrak{B}}, Y_{\mathfrak{B}}\right) \quad$ (for all $\left.X, Y \in \mathfrak{b}\right)$.

Proof. The implication $(b) \Rightarrow(a)$ is trivial. To prove that (a) implies (b), let $T \in$ $\mathfrak{L}(X, Y)$ and $B=\Gamma B \in \mathfrak{B}(X)$. Then $J_{B} \in L^{x}\left(X(B), X^{\mathfrak{B}}\right) \cap \mathfrak{L}(X(B), X)$, hence

$$
T J_{B}=T J_{B} i d_{X(B)} \in L^{x}\left(X(B), Y^{\mathfrak{B}}\right)
$$

[by (a)], thus $T \in L^{x}\left(X^{\mathfrak{B}}, Y^{\mathfrak{B}}\right)\left[\right.$ since $\left.T B \subseteq T\left(U_{X(B)}\right) \in \mathfrak{B}(Y)\right]$.

Definition 4.7 4.7. For any $Y \in \mathfrak{b}$, let $\mathfrak{B}(Y)$ be a separated convex bornology on $Y$ [which may be different from $\mathfrak{U}_{v o n}(Y)$ ]. The collection, defined by

$$
\mathfrak{B}=\{\mathfrak{B}(Y): Y \in \mathfrak{b}\}
$$

is called a generating bornology on $\mathfrak{b}$ is it satisfies the following two conditions:

(GB1) $\mathfrak{B}(Y) \subseteq \mathfrak{U}_{\text {von }}(Y)($ for all $Y \in \mathfrak{b})$;

(GB2) $\mathfrak{L}(X, Y) \subseteq L^{x}\left(X^{\mathfrak{B}}, Y^{\mathfrak{B}}\right)($ for all $X, Y \in \mathfrak{b})$

A generating bornology $\mathfrak{B}$ on $\mathfrak{b}$ is said to be $\sigma$-disked if on each $Y \in \mathfrak{b}$, the convex bornology $\mathfrak{B}(Y)$ admits a base consisting of $\sigma$-disked sets.

Remark(i). In[10], Stephani defines the concept of generating system of sets on Banach spaces. One can find that every generating bornology on $\mathbb{B}$ is a generating system of sets. However, we consider the current machinery be richer in both the representation 
and universal satisfication in arbitrary class of locally convex spaces. Moreover, interested readers may find that this two processes are at least equally powerful in the case of Banach spaces.

Remark(ii). As $\mathbb{K} \in \mathfrak{b}$, it follows that $\mathfrak{B}(\mathbb{K})=\mathfrak{U}_{\text {von }}(\mathbb{K})$, and hence from (GB2) that $\mathfrak{U}_{f}(Y) \subseteq \mathfrak{B}(Y) \subseteq \mathfrak{U}_{\text {von }}(Y)$ for any $Y \in \mathfrak{b}$.

Let $\mathfrak{B}$ be a generating bornology on $\mathfrak{b}$. Then (4.6) and (4.7) show that the class $\mathfrak{O}[\mathfrak{B}]$ of operators, defined by

$$
\mathfrak{O}[\mathfrak{B}](X, Y)=L^{x}\left(X, Y^{\mathfrak{B}}\right) \cap \mathfrak{L}(X, Y) \quad(\text { for all } X, Y \in \mathfrak{b})
$$

is an operator ideal on $\mathfrak{b}$; moreover, it is surjective as shown by the following result, which is a dual version of Lemma 3.7 .

Lemma 4.8 Let $\mathfrak{B}$ be a generating bornology on $\mathfrak{b}$. Then the class $\mathfrak{O}[\mathfrak{B}]$ of operators, defined by

$$
\mathfrak{O}[\mathfrak{B}](X, Y)=L^{x}\left(X, Y^{\mathfrak{B}}\right) \cap \mathfrak{L}(X, Y) \quad(\text { for all } X, Y \in \mathfrak{b}),
$$

is a surjective operator ideal on $\mathfrak{b}$, it is called the surjective operator ideal associated with $\mathfrak{B}$.

Proof. Suppose that $T \in \mathfrak{L}(X, Y), G \in \mathfrak{b}$ and that $Q: G \rightarrow X$ is an open operator such that $T Q \in \mathfrak{O}[\mathfrak{B}](G, Y)$. It is required to show that $T \in \mathfrak{O}[\mathfrak{B}](X, Y)(=$ $\left.L^{x}\left(X, Y^{\mathfrak{B}}\right) \cap \mathfrak{L}(X, Y)\right)$.

In fact, let $B \in \mathfrak{U}_{\text {von }}(X)$. As $\mathrm{Q}$ is open and continuous, there exists an $D \in \mathfrak{U}_{\text {von }}(G)$ such that $B \subseteq Q(D)$, hence

$$
T(B) \subseteq T(Q(D)) \in \mathfrak{B}(Y)
$$

by the assumption $T Q \in \mathfrak{O}[\mathfrak{B}](G, Y)=L^{x}\left(G, Y^{\mathfrak{B}}\right) \cap \mathfrak{L}(G, Y)$; this proves our assertion.

Theorem 4.9 (a) Given an operator ideal $\mathfrak{A}$ on $\mathfrak{b}$. The $\mathfrak{A}$-bornology $\mathfrak{U}(\mathfrak{A})$ on $\mathfrak{b}$ is a generating bornology on $\mathfrak{b}$. If, in addition, $\mathfrak{b}=\mathbb{B}$, then the surjective operator ideal $\mathfrak{O}[\mathfrak{U}(\mathfrak{A})]$ associated with $\mathfrak{U}(\mathfrak{A})$ is the surjective hull of $\mathfrak{A}$, that is,

$$
\mathfrak{O}[\mathfrak{U}(\mathfrak{A})]=\mathfrak{A}^{s u j} .
$$

Unfortunately, the preceeding equality is no longer true in the case of operator ideals on $\mathbb{L}$ by the same counterexample given in Theorem 3.8(a).

(b) Given a generating bornology $\mathfrak{B}$ on $\mathfrak{b}$, let $\mathfrak{O}[\mathfrak{B}]$ be the surjective operator ideal on 
$\mathfrak{b}$ associated with $\mathfrak{B}$. Then $\mathfrak{B}$ is the $\mathfrak{O}[\mathfrak{B}]$-bornology on $\mathfrak{b}$, that is,

$$
\mathfrak{B}=\mathfrak{U}(\mathfrak{O}[\mathfrak{B}]) .
$$

Consequently, every generating bornology on $\mathfrak{b}$ is an ideal-bornology on $\mathfrak{b}$.

Proof. (a)We check the condition (GB2) only. In fact, let B be an $\mathfrak{A}$-bounded set in $\mathrm{X}$ and $T \in \mathfrak{L}(X, Y)$ where $X, Y \in \mathfrak{C}$. By definition, there exists an $X_{0} \in \mathfrak{b}$ and $R \in \mathfrak{L}\left(X_{0}, X\right)$ such that $B \subseteq R B_{0}$ for some bounded set $B_{0}$ in $X_{0}$. Now $T B \subseteq T R B_{0}$ and $T R \in \mathfrak{A}\left(X_{0}, Y\right)$ give out our assertion that $T \in L^{x}\left(X^{\mathfrak{U}(\mathfrak{A})}, Y^{\mathfrak{U}(\mathfrak{A})}\right)$. If, in addition, $\mathfrak{b}=\mathbb{B}$, then $\mathrm{B}$ may be assumed to be $U_{x}$ and $B_{0}$ to be $U_{x_{0}}$. The equality follows from Lemma 2.1(b).

On each $Y \in \mathfrak{b}$, the ideal-bornology $\mathfrak{U}(\mathfrak{O}[\mathfrak{B}])$ on $\mathrm{Y}$ is the finest convex gornology on Y for which all operators in $\mathfrak{O}[\mathfrak{B}](X, Y)$ belong to $L^{x}\left(X, Y^{\mathfrak{U}(\mathfrak{O}[\mathfrak{B}])}\right)$ (whenever $\left.X \in \mathfrak{b}\right)$, it then follows from $\mathfrak{O}[\mathfrak{B}](X, Y)=L^{x}\left(X, Y^{\mathfrak{B}}\right) \cap \mathfrak{L}(X, Y)$ that $\mathfrak{B}(Y) \leqq \mathfrak{U}(\mathfrak{O}[\mathfrak{B}])(Y)$ or, equivalently,

$$
\mathfrak{U}(\mathfrak{O}[\mathfrak{B}])(Y) \subseteq \mathfrak{B}(Y) .
$$

Conversely, let $B=\Gamma B$ be in $\mathfrak{B}(Y)$. Then $J_{B}:\left(Y(B), r_{B}\right) \rightarrow Y$ is continuous [since $\left.\mathfrak{B}(Y) \subseteq \mathfrak{U}_{\text {von }}(Y)\right]$ and $J_{B}\left(U_{Y(B)}\right) \in \mathfrak{B}(Y)\left[\right.$ since $J_{B}\left(U_{Y(B)}\right) \subseteq 2 B$ and $\left.2 B \in \mathfrak{B}(Y)\right]$, thus

$$
J_{B} \in L^{x}\left(Y(B), Y^{\mathfrak{B}}\right) \cap \mathfrak{L}(Y(B), Y)=\mathfrak{O}[\mathfrak{B}](Y(B), Y) .
$$

As $\mathfrak{O}[\mathfrak{B}]$ is surjective (see Lemma 4.8), it follows from Theorem 4.5 that $\mathrm{B}$ is $\mathfrak{O}[\mathfrak{B}]$ bounded; this proves $\mathfrak{B}(Y) \subseteq \mathfrak{O}[\mathfrak{B}](Y)$.

We summarize the above discussion by the following result which is a dual version of Theorem 3.9.

Theorem 4.10 Let $\mathfrak{b}$ be either $\mathbb{L}$ or $\mathbb{B}$ and let

$$
\mathfrak{B}=\{\mathfrak{B}(Y): Y \in \mathfrak{b}\}
$$

be a family of separated convex (resp. $\sigma$-disked) bornologies on each locally convex space (resp. B-space). Then the following statements are equivalent.

(a) $\mathfrak{B}$ is an $\mathfrak{A}$-bornology on $\mathfrak{b}$.

(b) $\mathfrak{B}$ is a generating bornology on $\mathfrak{b}$.

(c) The set, defined by

$$
\mathfrak{O}[\mathfrak{B}](X, Y)=L^{x}\left(X, Y^{\mathfrak{B}}\right) \cap \mathfrak{L}(X, Y) \quad(X, Y \in \mathfrak{b}),
$$

is a surjective operator ideal on $\mathfrak{b}$. 


\section{A-Topological Spaces and $\mathfrak{A}$-bornological Spaces}

In terms of the notions of $\mathfrak{A}$-topologies and $\mathfrak{A}$-bornologies, we are able to make a classification of locally convex spaces as follows.

Definition 5.1 Let $\mathfrak{b}$ be either $\mathbb{B}$ or $\mathbb{L}$ and $\mathfrak{A}$ an operator ideal on $\mathfrak{b}$. An $X \in \mathfrak{b}$ is called an $\mathfrak{A}$-topological space(resp. $\mathfrak{A}$-bornological space) if the original topology (resp. $\mathfrak{A}$-bornology) on X.

By Lemma 3.3(resp. Lemma4.4), a Banach space E is an A-topological space(resp. $\mathfrak{A}$-bornological space) is and only if the quotient (resp. embedding) map associated with each continuous seminorm on E(resp. the $\sigma$-disked hull of each bounded set in E) belongs to the injective (resp. surjective) hull of $\mathfrak{A}$. Moreover, if we assume that the operator ideal $\mathfrak{A}$ on $\mathbb{L}$ is injective(resp.surjective), then Theorem 3.4(resp. Theorem4.5) shows that a locally convex space $\mathrm{X}$ is an $\mathfrak{A}$-topological space (resp. $\mathfrak{A}$-bornological space) is and only if the quotient(resp. embedding) map associated with each continuous seminorm on X (resp. absolutely convex, bounded set in X) belong to $\mathfrak{A}$.

Before giving some examples of $\mathfrak{A}$-topological spaces and $\mathfrak{A}$-bornological spaces, we recall the following terminologies. An operator $\mathrm{T}$ from a $\mathrm{B}$-space $\mathrm{E}$ into another $\mathrm{F}$ is said to be:

(a) compact(resp.weakly compact) if $T U_{E}$ is relatively compact (resp. relatively weakly compact);

(b) unconditionally summing if $\mathrm{T}$ sends each weakly unconditionally summable series in E onto a norm summable series;

(c) complete continuous if $\mathrm{T}$ sends each weak convergent sequence in $\mathrm{E}$ onto a norm convergent sequence in $\mathrm{F}$;

(d) separable if TE is separable;

(e) Banach-Saks(resp.weakly Banach-saks) if T sends each bounded (resp.weakly hull) sequence in $\mathrm{E}$ onto a sequence in $\mathrm{F}$ containing a subsequence with an convergent arithmetic mean;

(f) Rosenthal compact if $\mathrm{T}$ sends each bounded sequence in $\mathrm{E}$ onto a sequence in $\mathrm{F}$ containing a weakly Cauchy subsequence; 
(g) limited if $T U_{E}$ is a limit set in $\mathrm{F}$ (see [2]).

An operator $\mathrm{T}$ from a locally convex space $\mathrm{X}$ into another $\mathrm{Y}$ is said to be:

(h) precompact if $\mathrm{T}$ sends some O-neighbourhood in $\mathrm{X}$ into a precompact set in $\mathrm{Y}$;

(i) absolutely summing if for any continuous seminorm q on $\mathrm{Y}$ there are a $\sigma\left(X^{\prime}, X\right)$ closed equicontinuous subset $\mathrm{B}$ of $X^{\prime}$ and a positive Radon measure $\mu$ on B such that

$$
q(T x) \leqq \int_{B}\left|<x, x^{\prime}>\right| d \mu\left(x^{\prime}\right) \quad \text { for all } x \in X ;
$$

(j) of type $s$ if $\{T x: p(x) \leqq 1\}$ is bounded in $\mathrm{Y}$ for some strong nuclear seminorm $\mathrm{p}$ (see[8,p.97]).

We denote by $\mathfrak{F}$ for the ideal of continuous finite rank operators, by $\mathfrak{K}$ for the ideal of compact operators, by $\mathfrak{W}$ for the ideal of weakly compact operators, by $\mathfrak{U}_{P}$ for the ideal of unconditionally summing operators, by $\mathfrak{V}$ for the ideal of completely continuous operators, by $\mathfrak{X}$ for the ideal of separable operators, by $\mathfrak{B S}$ for the ideal of Banach-Saks operators, by $w \mathfrak{B G}$ for the ideal of weakly Banach-Saks operators, by $\mathfrak{R}$ for the ideal of Rosenthal compact operators, by $\mathfrak{L}$ im for the ideal of limited operators, by $\mathfrak{K}_{P}$ for the ideal of precompact operators, by $\mathfrak{P}$ for the ideal of absolutely summing operators and by $\mathfrak{N}_{S}$ for the ideal of operators of type s.

Example 5.2 5.2. (a) A Banach space E is a

(i) $\mathfrak{F}$-topological space (or $\mathfrak{K}$-topological space) if and only if $\operatorname{dim} E<\infty$;

(ii) $\mathfrak{A}_{P}$-topological space if and only if $\mathrm{E}$ has the Pelezynski property(i.e. E contains no copy of $\left.c_{0}\right)$;

(iii) $\mathfrak{B}$-topological space if and only if $\mathrm{E}$ has the Schur property(i.e. every weakly convergent sequence in $\mathrm{E}$ is norm convergent);

(iv) $\mathfrak{W}$-topological space if and only if $\mathrm{E}$ is reflexive;

(v) $\mathfrak{X}$-topological space if and only if E is separable;

(vi) $\mathfrak{W}^{-1} \circ \mathfrak{B}$-topological space if and only if $\mathrm{E}$ has the Dunford-Pettis property (i.e. every weakly compact operator from E into another Banach space F is completely continuous). 
(b) A locally convex space $\mathrm{X}$ is a

(i) $\mathfrak{K}_{P}$-topological space if and only if $\mathrm{X}$ is Schwartz;

(ii) $\mathfrak{P}$-topological space if and only if $\mathrm{X}$ is nuclear;

(iii) $\mathfrak{N}_{S^{-}}$-topological space if and only if $\mathrm{X}$ is strongly nuclear.

Proof. Being a Banach space, $\mathrm{E}$ is an $\mathfrak{A}$-topological space if and only if the identity map $i d_{E}$ belongs to $\mathfrak{A}^{i n j}(E, E)$. Now, (a)(i),(iii),(iv),(v), and(vi) are obvious. For (ii), we refer to Pietsch[7,p.48]. Moreover, (b)(i)and(iii) can be found in [8,(3.1) and (2.5)], while (b)(ii) is given in [13,p.46].

Example 5.3 5.3. (a)A Banach space E is a

(i) $\mathfrak{B S}$-bornological space if and only if E has the Banach-Saks property(i.e. every bounded sequence has a subsequence with convergent arithmetic mean);

(ii) $w \mathfrak{B} \mathfrak{S}$-bornological space if and only if $\mathrm{E}$ has the weak Banach-Saks property(i.e. every weakly null sequence has a subsequence with convergent arithmetic mean);

(iii) $\mathfrak{R}$-bornological space if and only if $\mathrm{E}$ has the Rosenthal property(i.e. E contains no copy of $\left.l_{1}\right)$;

(iv) $\mathfrak{K}-\mathfrak{L} \mathrm{im}^{-1}$-bornological space if and only if $\mathrm{E}$ has the Gelfand-Phillips property(i.e. every limited set in $\mathrm{E}$ is relatively norm compact).

(b)A locally convex space $\mathrm{X}$ is a

(i) $\mathfrak{K}$-bornological space if and only if $\mathrm{X}$ is semi-Montel;

(ii) $\mathfrak{W}$-bornological space if and only if $\mathrm{X}$ is semi-reflexive.

Proof. Similar to the proof of the preceeding example, a Banach space E is an $\mathfrak{A}$ bornological space if and only if the identity map $i d_{E}$ belongs to $\mathfrak{A}^{\operatorname{sur}}(E, E)$. Now, (a)(i) and (ii) are direct consequences of definitions. (a)(iii) is just the well-known Rosenthal theorem(see,[1,p.201]). (a)(iv) can be found in [2]. Recall that a locally convex space $\mathrm{X}$ is semi-Montel(resp. semi-reflexive) if and only if every bounded set in $\mathrm{X}$ is relative compact(resp. relatively weakly compact). It is now clear that (b)(i) and (ii) follow directly. 
The remainder of this section is devoted to establish some duality results between $\mathfrak{A}$-topological spaces and $\mathfrak{A}$-bornological spaces and $\mathfrak{A}$-bornological spaces, where $\mathfrak{A}$ is an operator ideal on $B$-spaces. To do this, we first recall the following terminology. Let $\mathfrak{A}$ be an operator ideal on B-spaces and for any $E, F \in \mathbb{B}$, let

$$
\mathfrak{A}^{\text {sur }}(E, F)=\left\{T \in \mathfrak{L}(E, F): T^{\prime} \in \mathfrak{A}\left(F^{\prime}, E^{\prime}\right)\right\}
$$

and

$$
\mathfrak{A}^{\text {reg }}(E, F)=\left\{T \in \mathfrak{L}(E, F): K_{F} T \in \mathfrak{A}\left(E, F^{\prime \prime}\right)\right\} .
$$

We say that $\mathfrak{A}$ is:

(a) symmetric if $\mathfrak{A} \subseteq \mathfrak{A}^{\text {dual }}$;

(b) completely symmetric if $\mathfrak{A}=\mathfrak{A}^{\text {dual }}$;

(c) regular if $\mathfrak{A}=\mathfrak{A}^{\text {reg }}$.

It is known from Pietsch [7,p.74] that

$$
\mathfrak{A}^{\text {injdual }}=\mathfrak{A}^{\text {dualsur }}, \quad \mathfrak{A}^{\text {surdual }} \supseteqq \mathfrak{A}^{\text {dualinj }}
$$

and from $[14$, p.801] that

$$
\mathfrak{A}^{\text {regsur }}=\mathfrak{A}^{\text {surreg }} .
$$

In the sequel, all polars dwelt on are absolutely polars. Polars taken with respect to the dual pair $\left\langle E, E^{\prime}>\right.$ are denoted by $M^{0}$, while polars taken with respect to $<E^{\prime}, E^{\prime \prime}>$ are denoted by $M^{\sharp}$, where $M$ is a set in one of the spaces $E, E^{\prime}$, and $E^{\prime \prime}$. In view of the definitions of generating bornologies and generating topologies, we have the following:

Theorem 5.4 (a)Let $\mathfrak{B}$ be a generating bornology on $B$-spaces. For any $F \in \mathbb{B}$, let

$$
\mathfrak{B}^{0}(F)=\left\{B^{0}: B \subset F^{\prime} \text { are } \mathfrak{B}\left(F^{\prime}\right)-\text { bounded }\right\}
$$

(it defines a topology on $F$ of uniform convergence on $\mathfrak{B}\left(F^{\prime}\right)$-bounded sets). Then the family

$$
\mathfrak{B}^{0}(F)=\left\{\mathfrak{B}^{0}(F): F \in \mathbb{B}\right\}
$$

is a generating topology on $B$-spaces, which is called the generating topology polar to $\mathfrak{B}$.

(b)Let $\mathfrak{g}$ be a generating topology on $B$-spaces. For any $E \in \mathbb{B}$, let

$$
\mathfrak{g}^{0}(E)=\left\{A: A \subset V_{0}, V \subset E^{\prime} \text { is convex } \mathfrak{g}\left(E^{\prime}\right)-\text { neighbourhoods of } 0\right\}
$$

(it defines an equicontinuous bornology on $E$ with respect to $\mathfrak{g}\left(E^{\prime}\right)$ ). Then the family

$$
\mathfrak{g}^{0}=\left\{\mathfrak{g}^{0}(E): E \in \mathbb{B}\right\}
$$


is a generating bornology on B-spaces, which is called the generating polar to $\mathfrak{g}$.

Proof.(a)The proof is similar to that given by Stephani[11,E6,p.244].

(b)We check (GB2) only. Let $T \in \mathfrak{L}(E, F)$ and $M \in \mathfrak{g}^{0}(E)$. Then $M \subseteq V^{0}$ for some closed and disked 0-neighbourhood $\mathrm{V}$ in $E_{\mathfrak{g}}^{\prime}$. Hence, $T M \subseteq T V^{0}$ and consequently

$$
(T M)^{0} \supseteqq\left(T V^{0}\right)^{0}=\left(T^{\prime}\right)^{-1} V
$$

by the bipolar theorem. Now, $\left(T^{\prime}\right)^{-1} V$ is a 0-neighbourhood in $F_{\mathfrak{g}}^{\prime}\left[\operatorname{since} T^{\prime} \in \mathfrak{L}\left(F^{\prime}, E^{\prime}\right)=\right.$ $\left.\mathfrak{L}\left(F_{\mathfrak{g}}^{\prime}, E_{\mathfrak{g}}^{\prime}\right)\right]$, this gives

$$
T M \subseteq(T M)^{00} \subseteq\left[\left(T^{\prime}\right)^{-1} V\right]^{0},
$$

that is, TM is bounded in $\left(F, \mathfrak{g}^{0}(F)\right)$, consequently,

$$
T \in L^{x}\left(E^{\mathfrak{g}^{0}(E)}, F^{\mathfrak{g}^{0}(F)}\right) .
$$

The most trivial examples may be the following:

$$
\mathfrak{g}_{\sigma}^{0}=\mathfrak{U}_{f}, \quad \mathfrak{U}_{f}^{0}=\mathfrak{g}_{\sigma}
$$

and

$$
\mathfrak{T}_{\text {ori }}^{0}=\mathfrak{U}_{\text {von }}^{0}, \quad \mathfrak{U}_{\text {von }}^{0}=\mathfrak{T}_{\text {ori }} .
$$

However, it is clear that $\mathfrak{g}^{00}(E)$ and $\mathfrak{B}^{00}(E)$ are, in general, not identical with $\mathfrak{g}(E)$ and $\mathfrak{B}(E)$ respectively unless $\mathrm{E}$ is reflexive. Nevertheless, it is quite appreciated if the underlying generating topology(resp. generating bornology) is an $\mathfrak{A}$-topology(resp. $\mathfrak{A}$ bornology) associated with some symmetric (resp.completely symmetric) operator ideal $\mathfrak{A}$ on $\mathbb{B}$.

Proposition 5.5 (Stephani[11,p.245]).Let $\mathfrak{A}$ be a symmetric operator ideal on B-spaces, let $\mathfrak{U}(\mathfrak{A})$ be the $\mathfrak{A}$-bornology on $\mathbb{B}$, and let $\mathfrak{U}(\mathfrak{A})^{0}$ be the generating topology polar to $\mathfrak{U}(\mathfrak{A})$. Then

$$
\mathfrak{O}\left[\mathfrak{U}(\mathfrak{A})^{0}\right]=\mathfrak{A}^{i n j} .
$$

Dually, we have the following:

Proposition 5.6 Let $\mathfrak{A}$ be a completely symmetric operator ideal on $B$-spaces, let $\mathfrak{P}(\mathfrak{A})$ be the $\mathfrak{A}$-topology on $\mathbb{B}$, and let $\mathfrak{P}(\mathfrak{A})^{0}$ be the generating bornology polar to $\mathfrak{P}(\mathfrak{A})$. Then

$$
\mathfrak{O}\left[\mathfrak{P}(\mathfrak{A})^{0}\right]=\mathfrak{A}^{\text {sur }} .
$$

Proof. Suppose that $T \in \mathfrak{A}^{\text {sur }}(E, F)$, where $E, F \in \mathbb{B}$. Then there exists an $G \in \mathbb{B}$ and an $R \in \mathfrak{A}(G, F)$ such that 


$$
T U_{E} \subseteq R U_{G}
$$

[see Lemma 2.1(b)], hence

$$
\left(T E_{E}\right)^{0} \supseteqq\left(R U_{G}\right)^{0}=\left(R^{\prime}\right)^{-1}\left(U_{G^{\prime}}\right) .
$$

As $\mathfrak{A}$ is completely symmetric, it follows that

$$
R^{\prime} \in \mathfrak{A}\left(F^{\prime}, G^{\prime}\right)
$$

and hence that $\left(R^{\prime}\right)^{-1}\left(U_{G^{\prime}}\right)$ is a $\mathfrak{P}(\mathfrak{A})$-neighbourhood of 0 in $F^{\prime}$. We then conclude from (1) that $\left(T U_{E}\right)^{0}$ is a $\mathfrak{P}(\mathfrak{A})$-neighbourhood of 0 in $F^{\prime}$; consequently, $T U_{E}$ is $\mathfrak{P}^{0}(F)$ bounded in $\mathrm{F}$ since $T U_{E} \subseteq\left(T U_{E}\right)^{00}$. This shows that $T \in L^{x}\left(E, F^{\mathfrak{P}(\mathfrak{A})^{0}}\right)$, and a fortiori, $T \in \mathfrak{O}\left[\mathfrak{P}(\mathfrak{A})^{0}\right](E, F)$.

Conversely, let $T \in \mathfrak{O}\left[\mathfrak{P}(\mathfrak{A})^{0}\right](E, F)$. Then $T U_{E}$ is bounded in the bornological $\operatorname{space}\left(F, \mathfrak{P}(\mathfrak{A})^{0}(F)\right)$, and hence there exists a colsed and disked 0-neighbourhood $\mathrm{V}$ in $\left(F^{\prime}, \mathfrak{P}(\mathfrak{A})\left(F^{\prime}\right)\right)$ such that $T U_{E} \subseteq V^{0}$. By the definition of $\mathfrak{A}$-topology, there exists an $G \in \mathbb{B}$ and $R \in \mathfrak{A}\left(F^{\prime}, G\right)$ such that $V \supseteqq R^{-1} U_{G}$. It then follows from

$$
(R V)^{0}=\left(R^{\prime}\right)^{-1}\left(V^{\sharp}\right) \subseteq U_{G^{\prime}}
$$

that

$$
K_{F} T U_{E} \subseteq K_{F} V^{0} \subseteq V^{\sharp} \subseteq R^{\prime} U_{G^{\prime}},
$$

and hence from Lemma 2.1(b) and $R^{\prime} \in \mathfrak{A}\left(G^{\prime}, F^{\prime \prime}\right)$ that

$$
K_{F} T \in \mathfrak{A}^{\text {sur }}\left(E, F^{\prime \prime}\right) .
$$

On the other hand, it is known (see[14]) that

$$
\left(\mathfrak{A}^{\text {sur }}\right)^{\text {reg }}=\left(\mathfrak{A}^{\text {reg }}\right)^{\text {sur }}
$$

for any operator ideal $\mathfrak{A}$ on B-spaces. Hence we obtain

$$
T \in \mathfrak{A}^{\text {surreg }}(E, F)=\mathfrak{A}^{\text {regsur }}(E, F)=\mathfrak{A}^{\text {sur }}(E, F)
$$

since $\mathfrak{A}$ is assumed to be completely symmetric and hence regular.

Theorem 5.7 Let $\mathfrak{A}$ be a symmetric operator ideal on $B$-spaces. Let $\mathfrak{P}(\mathfrak{A})$ be the $\mathfrak{A}$ topology on $\mathbb{B}$ and $\mathfrak{U}(\mathfrak{A})$ the $\mathfrak{A}$-bornology on $\mathbb{B}$. Then

$$
\mathfrak{U}(\mathfrak{A})^{0}=\mathfrak{P}(\mathfrak{A}) .
$$

Moreover, if $\mathfrak{A}$ is associated to be completely symmetric then

$$
\mathfrak{P}(\mathfrak{A})^{0}=\mathfrak{U}(\mathfrak{A}) .
$$

In this case, we also have

$$
\mathfrak{P}(\mathfrak{A})^{00}=\mathfrak{P}(\mathfrak{A}) \quad \text { and } \quad \mathfrak{U}(\mathfrak{A})^{00}=\mathfrak{U}(\mathfrak{A})
$$


Proof. Since

$$
\mathfrak{O}[\mathfrak{P}(\mathfrak{A})]=\mathfrak{P}(\mathfrak{A})^{i n j} \quad \text { and } \quad \mathfrak{O}[\mathfrak{U}(\mathfrak{A})]=\mathfrak{A}^{\text {sur }},
$$

it follows from propositions (5.5) and (5.6) that

$$
\mathfrak{O}[\mathfrak{P}(\mathfrak{A})]=\mathfrak{O}\left[\mathfrak{U}(\mathfrak{A})^{0}\right] \quad \text { and } \quad \mathfrak{O}[\mathfrak{U}(\mathfrak{A})]=\mathfrak{O}\left[\mathfrak{P}(\mathfrak{A})^{0}\right]
$$

and hence from Propositions 3.8(b) and 4.9(b) that

$$
\mathfrak{P}(\mathfrak{A})=\mathfrak{U}(\mathfrak{A})^{0} \quad \text { and } \quad \mathfrak{U}(\mathfrak{A})=\mathfrak{P}(\mathfrak{A})^{0} .
$$

Example 5.8 As the ideals $\mathfrak{K}$ and $\mathfrak{W}$ of compact and weakly compact operators are both completely symmetric and the ideal $\mathfrak{N}$ of nuclear operators is symmetric, we have

$$
\mathfrak{U}_{v}^{0}=\mathfrak{g}_{v}, \quad \mathfrak{U}_{c}^{0}=\mathfrak{g}_{p c}, \quad \mathfrak{g}_{p c}^{0}=\mathfrak{U}_{c}
$$

and

$$
\mathfrak{U}_{w c}^{0}=\mathfrak{g}_{w c}, \quad \mathfrak{g}_{w c}^{0}=\mathfrak{U}_{w c}
$$

where $\mathfrak{g}_{v}, \mathfrak{g}_{p c}$ and $\mathfrak{g}_{w c}\left(\right.$ resp. $\mathfrak{U}_{v}, \mathfrak{U}_{p c}$ and $\left.\mathfrak{U}_{w c}\right)$ are the generating systems of nuclear topologies, precompact topologies and weakly compact topologies (resp. nuclear bronologies, precompact bornologies and weakly compact bornologies), respectively. Note that $\mathfrak{g}_{p c}=\mathfrak{g}_{c}$ (the generating system of compact topologies) on $\mathbb{B}$.

The above equalities show that each of the three generating topologies is the polar topology induced by the corresponding system of bornologies. On the other hand, the compact bornologies and the weakly compact bornologies are the equicontinuous bornologies of the corresponding system of topologies.

The following concluding result is very desirable.

Theorem 5.9 Let $\mathfrak{A}$ be an operator ideal on B-space.

(a) If $\mathfrak{A}$ is symmetric, then a Banach space $E$ is an $\mathfrak{A}$-topological space if and only if its Banach dual $E^{\prime}$ is an $\mathfrak{A}$-bornological space.

(b) If $\mathfrak{A}$ is assumed to be completely symmetric, then a Banach space $E$ is an $\mathfrak{A}$ bornological space if and only if $E^{\prime}$ is an $\mathfrak{A}$-topological space.

Proof.(a)The assertion follows from the following equivalent statements:

$\mathrm{E}$ is an $\mathfrak{A}$-bornological space

$$
\begin{aligned}
& \Leftrightarrow \mathfrak{U}(\mathfrak{A})\left(E^{\prime}\right)=\mathfrak{U}_{\text {von }}\left(E^{\prime}\right) \\
& \Leftrightarrow \mathfrak{U}(\mathfrak{A})^{0}(E)=\|\cdot\|_{E^{-} \text {topology }} \\
& \Leftrightarrow \mathfrak{P}(\mathfrak{A})(E)=\|\cdot\|_{E^{-} \text {-topology }} \\
& \Leftrightarrow E \text { is an } \mathfrak{A} \text {-topological space. }
\end{aligned}
$$


(b)Follows from the following equivalent statements:

$$
\begin{aligned}
E^{\prime} & \text { is an } \mathfrak{A} \text {-topological space } \\
& \Leftrightarrow \mathfrak{P}(\mathfrak{A})\left(E^{\prime}\right)=\|\cdot\|_{E^{-} \text {topology }} \\
& \Leftrightarrow \mathfrak{P}(\mathfrak{A})^{0}(E)=\mathfrak{U}_{\text {von }}(E) \\
& \Leftrightarrow \mathfrak{U}(\mathfrak{A})(E)=\mathfrak{U}_{\text {von }}(E) \\
& \Leftrightarrow \mathrm{E} \text { is an } \mathfrak{A} \text {-bornological space. }
\end{aligned}
$$

Trivial examples can be given by those properties determined by the ideals $\mathfrak{F}, \mathfrak{W}$, and $\mathfrak{H}$ of continuous finite rank operators, weakly compact operators and Hilbert operators, respectively[where $T \in \mathfrak{L}(E, F)$ is called a Hilbert operator if $\mathrm{T}$ can be factorized through a Hilbert space]. Namely, for a B-space E, we have

(a) $\mathrm{E}$ is of finite dimensional if and only if $E^{\prime}$ is of finite dimensional.

(b) $\mathrm{E}$ is reflexive if and only if $E^{\prime}$ is reflexive.

(c) $\mathrm{E}$ is a Hilbert space if and only if $E^{\prime}$ is a Hilbert space.

\section{$6 \mathfrak{O}[\mathfrak{g} / \mathfrak{B}]$-Operator Ideals and $\mathfrak{O}[\mathfrak{g} / \mathfrak{B}]$-Spaces}

In this final section, on the well-paved background due to the several preceeding sections, we are able to define the most natural and the most applicable type of operator ideals on $\mathbb{L}$, i.e. the $\mathfrak{O}[\mathfrak{g} / \mathfrak{B}]$-operator ideals.

Some original of this section should be referred to Randtke[8] and Wong[12,pp.142,148] and $[13, p p .24,156,163]$. The key point of our theory may be that the topological and the locally convex space can be highlighted by the behaviour of the continuous seminorm system of this space. Moreover, the family of quotient mappings associated to every continuous seminorm on a locally convex space contains rich information on the structure of this space, too. By this principle, we express most of the classical examples given in the above books with the terminology of $\mathfrak{O}[\mathfrak{g} / \mathfrak{B}]$-spaces.

Readers should be noticed that our presentation of $\mathfrak{O}[\mathfrak{g} / \mathfrak{B}]$-spaces is a method to study LCS's with the tool of operator ideal similar to the one employed by Grothendieck and Pietsch(cf. the last chapter of the book of Pietsch[7]). But, we consider our method more natural and easier to follow than the Pietsch's one.

Definition 6.1 6.1. Let $\mathfrak{b}$ be either $\mathbb{L}$ or $\mathbb{B}$. Let $\mathfrak{g}$ be a generating topology on $\mathfrak{b}$, let $\mathfrak{B}$ be a generating bornology on $\mathfrak{b}$ and suppose for any $X, Y \in \mathfrak{b}$ that 


$$
\mathfrak{O}[\mathfrak{g} / \mathfrak{B}](X, Y)=\mathfrak{L}^{b}\left(X_{\mathfrak{g}}, Y^{\mathfrak{B}}\right)
$$

denotes the class of all bounded operators from $X_{\mathfrak{g}}=(X, \mathfrak{g}(X))$ into the bornological space $Y^{\mathfrak{B}}=(Y, \mathfrak{B}(Y))$ (i.e. $\left.T \in \mathfrak{L}^{b}\left(X_{\mathfrak{g}}, Y^{\mathfrak{B}}\right)\right)$ if $\mathrm{T}$ sends some 0-neighbourhood in $X_{\mathfrak{g}}$ onto a bounded set in $Y^{\mathfrak{B}}$. Members in $\mathfrak{O}[\mathfrak{g} / \mathfrak{B}]$ are called $\mathfrak{g}$ - $\mathfrak{B}$-bounded operators.

By the definitions of generating topologies and generating bornologies, it is easily seen that

$$
\mathfrak{O}[\mathfrak{g} / \mathfrak{B}](X, Y) \subseteq \mathfrak{L}\left(X_{\mathfrak{g}}, Y\right) \quad \text { for all } X, Y \in \mathfrak{b}
$$

and that an $T \in L(X, Y)$ is $\mathfrak{g}$ - $\mathfrak{B}$-bounded if and only if there exists a $\mathfrak{g}(X)$-continuous seminorm $\mathrm{p}$ on $\mathrm{X}$ such that

$$
\{T x \in Y: p(x) \leqq 1\}
$$

is $\mathfrak{B}(Y)$-bounded in $\mathrm{Y}$. Thus, the notion of $\mathfrak{g}$ - $\mathfrak{B}$-bounded operators is a generalization of that of precompact-bounded (i.e., quasi-Schwartz operators in the terminology of Randtke [8])(resp. prenuclear-bounded, quasi-nuclear-bounded and cone-prenuclear maps) operators of Wong[13, pp.24,156, and 163] and [12, p.142].

Theorem 6.2 Let $\mathfrak{b}$ be either $\mathbb{L}$ or $\mathbb{B}$, let $\mathfrak{g}$ be a generating topology on $\mathfrak{b}$ and $\mathfrak{B}$ a generating bornology on $\mathfrak{b}$. Then $\mathfrak{O}[\mathfrak{g} / \mathfrak{B}]$ is an operator ideal on $\mathfrak{b}$.

Proof. The condition $\left(O I_{0}\right)$ is obvious. For $\left(O I_{0}\right)$, let $T_{1}$ and $T_{2} \in \mathfrak{O}[\mathfrak{g} / \mathfrak{B}](X, Y)$ for some $X, Y \in \mathfrak{b}$. Then there are two $\mathfrak{g}(X)$-neighbourhood $U_{1}$ and $U_{2}$ of 0 in $\mathrm{X}$ such that $M_{1}=T_{1} U_{1}$ and $M_{2}=T_{2} U_{2}$ are both bounded in $Y^{\mathfrak{B}}$. Let $U$ be any $\mathfrak{g}(X)$-neighbourhood of 0 in X such that $U \subseteq U_{1}$ and $U \subseteq U_{2}$. Then the set $\left(T_{1}+T_{2}\right) U \subseteq M_{1}+M_{2}$ is clearly bounded in $Y^{\mathfrak{B}}$ and hence $\left(O I_{1}\right)$ follows.

Finally, to check that $\mathfrak{O}[\mathfrak{g} / \mathfrak{B}]$ satisfies the condition $\left(O I_{2}\right)$, suppose that $X_{0}, X, Y$, $Y_{0} \in \mathfrak{b}$ and that

$$
R \in \mathfrak{L}\left(Y, Y_{0}\right), \quad T \in \mathfrak{O}[\mathfrak{g} / \mathfrak{B}](X, Y) \quad \text { and } \quad S \in \mathfrak{L}\left(X_{0}, X\right) .
$$

As $T \in \mathfrak{O}[\mathfrak{g} / \mathfrak{B}](X, Y)\left(=\mathfrak{L}^{b}\left(X_{\mathfrak{g}}, Y^{\mathfrak{B}}\right)\right)$, it follows that there is a $\mathfrak{g}(X)$-neighbourhood $\mathrm{V}$ of 0 in $\mathrm{X}$ such that $T(V)$ is $\mathfrak{B}(Y)$-bounded in $\mathrm{Y}$, and hence from $\left(G T_{2}\right)$ [i.e., $S \in$ $\left.\mathfrak{L}\left(X_{0}, X\right) \subseteq \mathfrak{L}\left(\left(X_{0}\right)_{\mathfrak{g}}, X_{\mathfrak{g}}\right)\right]$ that there is a $\mathfrak{g}(X)$-neighbourhood $V_{0}$ of 0 in $X_{0}$ such that $S V_{0} \subseteq V$, and thus $T S V_{0}$ is $\mathfrak{B}(Y)$-bounded in $\mathrm{Y}$ [since $T S V_{0} \subseteq T V \in \mathfrak{B}(Y)$ ]. On the other hand, since $\mathfrak{B}$ is assumed to be a generating bornology, it follows from $\left(G B_{2}\right)$ [i.e. $\left.R \in \mathfrak{L}\left(Y, Y_{0}\right) \subseteq L^{x}\left(Y^{\mathfrak{B}}, Y_{0}^{\mathfrak{B}}\right)\right]$ and $T S V_{0} \in \mathfrak{B}(Y)$ that $(R T S) V_{0}$ is $\mathfrak{B}(Y)$-bounded in $Y_{0}$. This implies that $R T S \in \mathfrak{L}^{b}\left(\left(X_{0}\right)_{\mathfrak{g}}, Y_{0}^{\mathfrak{B}}\right)=\mathfrak{O}[\mathfrak{g} / \mathfrak{B}]\left(X_{0}, Y_{0}\right)$.

It is natural to ask under what conditions on $\mathfrak{b}$ and $\mathfrak{B}$, the operator ideal $\mathfrak{O}[\mathfrak{g} / \mathfrak{B}]$ is injective or surjective. The following two results, which are dual to each other, give 
such sufficient conditions.

Proposition 6.3 Let $\mathfrak{b}$ be either $\mathbb{L}$ or $\mathbb{B}$ and $\mathfrak{B}$ a generating bornology on $\mathfrak{b}$. If $\mathfrak{B}$ satisfies the following condition:

(Bor)for any $Y, Y_{0} \in \mathfrak{b}$, if $J: Y \rightarrow Y_{0}$ is a topological injection then

$$
J^{-1}\left(\mathfrak{B}\left(Y_{0}\right)\right)=\left\{J^{-1} B: B \in \mathfrak{B}\left(Y_{0}\right)\right\}
$$

is a base for $\mathfrak{B}(Y)$,

then the operator ideal $\mathfrak{O}[\mathfrak{g} / \mathfrak{B}]$ on $\mathfrak{b}$ is injective whenever $\mathfrak{g}$ is any generating topology on $\mathfrak{b}$.

Proof. Let $T \in \mathfrak{L}(X, Y)$ and let $J: Y \rightarrow Y_{0}$ be a topological injection such that $J T \in \mathfrak{O}[\mathfrak{g} / \mathfrak{B}]\left(X, Y_{0}\right)$ (where $\left.X, Y, Y_{0} \in \mathfrak{b}\right)$. Then the definition of $\mathfrak{O}[\mathfrak{g} / \mathfrak{B}]$ ensures that there exists a $\mathfrak{g}(X)$-neighbourhood $\mathrm{V}$ of 0 in $\mathrm{X}$ such that $J T(V)$ is $\mathfrak{B}(Y)$-bounded in $Y_{0}$. Now the condition (Bor), together with $J^{-1}(J T(V))=T(V)$, implies that $T(V)$ is $\mathfrak{B}(Y)$-bounded in $Y$; this shows that $T \in \mathfrak{O}[\mathfrak{g} / \mathfrak{B}](X, Y)$, and thus $\mathfrak{O}[\mathfrak{g} / \mathfrak{B}]$ is injective.

Proposition 6.4 Let $\mathfrak{b}$ be either $\mathbb{L}$ or $\mathbb{B}$ and $\mathfrak{g}$ a generating topology on $\mathfrak{b}$. If $\mathfrak{g}$ satisfies the following condition:

(Top) for any $X, X_{0} \in \mathfrak{b}$, if $Q: X_{0} \rightarrow X$ is an open operator then $Q:\left(X_{0}, \mathfrak{g}\left(X_{0}\right)\right) \rightarrow$ $(X, \mathfrak{g}(X))$ is open and continuous:

then the operator ideal $\mathfrak{O}[\mathfrak{g} / \mathfrak{B}]$ on $\mathfrak{b}$ is surjective whenever $\mathfrak{B}$ is any generating bornology on $\mathfrak{b}$.

Proof. Let $T \in \mathfrak{L}(X, Y)$ and let $Q: X_{0} \rightarrow X$ be an operator such that $T Q \in$ $\mathfrak{O}[\mathfrak{g} / \mathfrak{B}]\left(X_{0}, Y\right)$ (where $\left.X_{0}, X, Y \in \mathfrak{b}\right)$. Then there exists a $\mathfrak{g}\left(X_{0}\right)$-neighbourhood $V_{0}$ in $X_{0}$ such that $T Q\left(V_{0}\right)$ is $\mathfrak{B}(Y)$-bounded in Y. By the condition (Top), $V=Q\left(V_{0}\right)$ is $\mathfrak{g}(Y)$-neighbourhood of 0 in $Y$, hence $T \in \mathfrak{O}[\mathfrak{g} / \mathfrak{B}](X, Y)$, and thus $\mathfrak{O}[\mathfrak{g} / \mathfrak{B}]$ is surjective.

Now we consider two special cases of $\mathfrak{O}[\mathfrak{g} / \mathfrak{B}]$-operator ideals, namely the one $\mathfrak{g}=$ $\mathfrak{T}_{\text {ori }}$ (the original topologies) and the one $\mathfrak{B}=\mathfrak{U}_{\text {von }}$. They seem to be the most popular (if not unique) cases occupying general interests. For simplicity of notations, we denoted by $\mathfrak{O}^{b}[\mathfrak{g}]$ and $\mathfrak{O}^{b}[\mathfrak{B}]$ for the ideals $\mathfrak{O}\left[\mathfrak{g} / \mathfrak{U}_{\text {von }}\right]$ and $\mathfrak{O}\left[\mathfrak{T}_{\text {ori }} / \mathfrak{B}\right]$, respectively. As corollaries of Propositions (6.3) and (6.4), we see that $\mathfrak{O}^{b}[\mathfrak{g}]$ is injective while $\mathfrak{O}^{b}[\mathfrak{B}]$ is surjective for any generating topology $\mathfrak{g}$ and any generating bornology $\mathfrak{B}$.

By a similar argument given in the proofs of propositions (3.8)(b) and (4.9)(b), one 
can verify the following:

Proposition 6.5 Let $\mathfrak{g}$ be a generating topology and $\mathfrak{B}$ be a generating bornology on LCS's. Then we have

$$
\mathfrak{P}\left(\mathfrak{O}^{b}[\mathfrak{g}]\right)=\mathfrak{g} \quad \text { and } \quad \mathfrak{U}\left(\mathfrak{D}^{b}[\mathfrak{B}]\right)=\mathfrak{B} .
$$

As Stephani[10 and 11] proved the following elegant formulae

$$
\mathfrak{O}[\mathfrak{P}(\mathfrak{A})]=\mathfrak{A}^{\text {inj }} \quad \text { and } \quad \mathfrak{O}[\mathfrak{U}(\mathfrak{A})]=\mathfrak{A}^{\text {sur }},
$$

where $\mathfrak{A}$ is an operator ideal on B-spaces, it might be hopeful to expect the following result.

$(*)$ For any bounded operator ideal $\mathfrak{A}$ on $\mathbb{L}$ we would have

$$
\mathfrak{O}^{b}[\mathfrak{P}(\mathfrak{A})]=\mathfrak{A}^{i n j} \text {. }
$$

Note that the procedures $\mathfrak{O}$ and $\mathfrak{O}^{b}$ are identical in $\mathbb{B}$ and it is easy to see that the formula $\mathfrak{O}[\mathfrak{P}(\mathfrak{A})]=\mathfrak{A}^{i n j}$ is generally invalid.

The statement $(*)$ is suggested by Franco and Piñeiro as a theorem in their paper[3,Theorem 1 in Sect.2]. Unfortunately, their proof of this statement is not true (see Example 6.6 below). They have overlooked an important fact that $\mathfrak{O}^{b}[\mathfrak{P}(\mathfrak{A})]$ needs not always contain $\mathfrak{A}$. In contrast, we have $\mathfrak{D}^{b}[\mathfrak{P}(\mathfrak{A})] \subseteq \mathfrak{A}$ for any injective operator ideal $\mathfrak{A}$.

Example 6.6 Taking $\mathfrak{A}=\mathfrak{K}_{p}$, the injective operator ideal of all precompact operators between LCS's. Then we have that $\mathfrak{P}\left(\mathfrak{K}_{p}\right)$, the generating system of precompact topologies on LCS's, and $\mathfrak{O}^{b}\left[\mathfrak{g}_{p c}\right]=\mathfrak{K}_{p}^{b}$, the ideal of all precompact-bounded operators(or quasi-Schwartz operators in the terminology of Randtke [8]) between LCS's. Now, by a result of Randtke[8](see also[13,pp.15,24,25, and 26]), $\mathfrak{K}_{p}^{b}(X, Y) \subseteq \mathfrak{K}(X, Y)$ for any locally convex spaces $\mathrm{X}$ and $\mathrm{Y}$, and the equality is, in general, not valid. In particular, for a fixed locally convex space $X, \mathfrak{K}_{p}(X, Y)=\mathfrak{K}_{p}^{b}(X, Y)$ for all locally convex spaces $\mathrm{Y}$ if and only if $\mathrm{X}$ is a Schwartz space. Thus statement $(*)$ is false.

We are now ready to give some most important examples all of which can be found in the book of Wong [13,pp.5, 163, and 156].

Example 6.7 (a)The ideal $\mathfrak{O}\left[\mathfrak{T}_{\text {ori }} / \mathfrak{U}_{\text {von }}\right]$ is the class $\mathfrak{L}^{b}$ of all bounded operators on $\mathbb{L}$, where $\mathfrak{T}_{\text {ori }}$ denotes the generating topology defined by the original topologies on all LCS's.

(b)The ideal $\mathfrak{O}\left[\mathfrak{T}_{\text {ori }} / \mathfrak{U}_{c}\right]$ is the class $\mathfrak{K}$ od all compact operators on $\mathbb{L}$, where $\mathfrak{U}_{c}$ denotes the generating bornology of compact bornologies. 
(c)The ideal $\mathfrak{O}\left[\mathfrak{T}_{\text {ori }} / \mathfrak{U}_{w c}\right]$ is the class $\mathfrak{W}$ of all weakly compact operators on $\mathbb{L}$, where $\mathfrak{U}_{w c}$ denotes the generating bornology of weakly compact bornologies.

(d)The ideal $\mathfrak{O}\left[\mathfrak{g}_{q v} / \mathfrak{U}_{\text {von }}\right]$ is the class $\mathfrak{N}_{q}^{b}$ of all quasi-nuclear-bounded operators on $\mathbb{L}$. where $\mathfrak{g}_{q v}$ denotes the generating topology of quasi-nuclear topologies.

(e)The ideal $\mathfrak{O}\left[\mathfrak{g}_{p v} / \mathfrak{U}_{\text {von }}\right]$ is the class $\mathfrak{N}_{p}^{b}$ of all prenuclear-bounded operators on $b l$, where $\mathfrak{g}_{p v}$ denotes the generating topology of prenuclear topologies.

(f)The ideal $\mathfrak{O}\left[\mathfrak{g}_{p c} / \mathfrak{U}_{\text {von }}\right]$ is the class $\mathfrak{K}_{p}^{b}$ of all precompact-bounded operators (or quasiSchwartz operators in terminology of Randtke [8])on $b l$, where $\mathfrak{g}_{p c}$ denotes the generating topology of precompact topologies.

(g)The ideal $\mathfrak{O}\left[\mathfrak{g}_{s v} / \mathfrak{U}_{\text {von }}\right]$ is the class $\mathfrak{N}_{s}^{b}$ of all strongly nuclear-bounded operators (or operators of type $s$, in terminology of Randlke, [8]) on $b l$, where $\mathfrak{g}_{s v}$ denotes the generating topology of strongly nuclear topologies.

By propositions(6.3) and (6.4), we see immediately that the ideals $\mathfrak{L}^{b}, \mathfrak{K}$, and $\mathfrak{W}$ are all surjective while the ideals $\mathfrak{L}^{b}, \mathfrak{N}_{q}^{b}, \mathfrak{N}_{p}^{b}, \mathfrak{K}_{p}^{b}$, and $\mathfrak{N}_{s}^{b}$ are all injective since $\mathfrak{T}_{\text {ori }}$ and $\mathfrak{U}_{\text {von }}$ enjoy the properties stated in Propositions (6.3) and (6.4), respectively.

Using the same machineries, one can introduce as many operator ideals by classifying their topological-bornological property as one likes. For example, the ideal $\mathfrak{O}\left[\mathfrak{g}_{p c} / \mathfrak{U}_{c}\right]$ is the class of all operators $T \in \mathfrak{L}(X, Y)$ with $X, Y \in \mathbb{L}$ such that for some precompact seminorm $\mathrm{p}$ on $\mathrm{X}$, the set $\{T x: p(x) \leqq 1\}$ is compact in $\mathrm{X}$. There seems to be a great deal of possibilities to introduce new classes of operators under this principle.

Finally, we introduce a concept which is well-known to be a very powerful tool in the theory of locally convex spaces.

Definition 6.8 Let $\mathfrak{g}$ be a generating topology on $\mathbb{L}$ and $\mathfrak{B}$ a generating bornology on $\mathbb{L}$. A locally convex space $X$ is called an $\mathfrak{O}[\mathfrak{g} / \mathfrak{B}]$-space if for any continuous seminorm p on $\mathrm{X}$, the quotient map $Q_{p}: X \rightarrow X / p^{-1}(0)$ belongs to $\mathfrak{O}[\mathfrak{g} / \mathfrak{B}]\left(X, X / p^{-1}(0)\right)$.

Theorem 6.9 For a locally convex space $X$, we have:

(a) $X$ is a Schwartz space if and only if $X$ is a $\mathfrak{K}_{p}^{b}$-space.

(b) $X$ is a nuclear space if and only if $X$ is a $\mathfrak{N}_{s}^{b}$-space.

(c) $X$ is a strongly nuclear space if and only if $X$ is a $\mathfrak{N}_{s}^{b}$-space.

Proof. For (a) see p.17, for (b) see p.164, and for (c) see p.226 of the book of Wong[13]. 
$\mathfrak{A}, \mathfrak{B}, \mathfrak{C}, \mathfrak{D}, \mathfrak{E}, \mathfrak{F}, \mathfrak{G}, \mathfrak{H}, \mathfrak{I}, \mathfrak{J}, \mathfrak{K}, \mathfrak{L}, \mathfrak{M}, \mathfrak{N}, \mathfrak{O}, \mathfrak{P}, \mathfrak{Q}, \mathfrak{R}, \mathfrak{S}, \mathfrak{T}, \mathfrak{U}, \mathfrak{V}, \mathfrak{W}, \mathfrak{X}, \mathfrak{Y}, \mathfrak{Z}$,

$\mathfrak{a}, \mathfrak{b}, \mathfrak{c}, \mathfrak{d}, \mathfrak{e}, \mathfrak{f}, \mathfrak{g}, \mathfrak{h}, \mathfrak{i}, \mathfrak{j}, \mathfrak{k}, \mathfrak{l}, \mathfrak{m}, \mathfrak{n}, \mathfrak{o}, \mathfrak{p}, \mathfrak{q}, \mathfrak{r}, \mathfrak{s}, \mathfrak{t}, \mathfrak{u}, \mathfrak{v}, \mathfrak{w}, \mathfrak{x}, \mathfrak{y}, \mathfrak{z}$

\section{References}

[1] Diestel,J.:Sequences and series in Banach spaces(GTM, Vol.92). Berlin Heidelberg New York:Spring 1984

[2] Drewnowski,L.:On Banach spaces with Gelfand-Phillips property.Math.Z.193,405411(1986)

[3] L. Franco and C. Piñeiro, "The injective hull of an operator ideal on locally convex spaces", Manuscripa Math., 38, 1982, 333-341.

[4] H. Hogbe-Nlend, "Bornologies and functional analysis", Math. Studies 26, NorthHolland, Amsterdam, 1977.

[5] Junk,H.:Locally convex spaces and operator ideals(Teubner-Texte zur Math.,Band 56). Leipzig:Teubner 1983

[6] Köthe,G.:Topological vector spaces, I.Berlin Heidelberg New York: Spring 1969

[7] Pietsch,A.:Operator ideals. Amsterdam: North-Holland 1980

[8] Randtke,D.:Characterizations of precompact maps, Schwartz spaces and nuclear spaces. Trans. Am. Math. Soc. 165,87-101(1972)

[9] Schaefer,H.H.:Topological vector spaces. Berlin Heidelberg New York: Springer 1971

[10] Stephani,I.:Generating topologies and quotients of surjective operator ideals. Math. Nachr. 99, 13-27

[11] Stephani,I.: "Generating topologies and quotients of injective operator ideals", in "Banach Space Theory and Its Application (proceedings, Bucharest 1981)", Lecture Notes in Math., vol. 991, Springer-Verlag, Berlin-Heidelberg-New York, 1983, 239255 . 
[12] Yau-chuen Wong: "The topology of uniform convergence on order-bounded sets", Lecture Notes in Mathematics, vol. 531, Springer-Verlag, Berlin-Heidelberg-New York, 1976.

[13] Yau-chuen Wong: "Schwartz spaces, nuclear spaces and tensor products", Lecture Notes in Mathematics, vol. 726, Springer-Verlag, Berlin-Heidelberg-New York, 1979.

[14] Yau-chuen Wong: On a formula of the regular hull of operator ideals. Math.Semin.Notes, Kobe Univ. 10, 797-802(1982) 